\title{
Finescale Clusterization Intermittency of Turbulence in the Atmospheric Boundary Layer
}

\author{
LEI LIU AND FEI HU \\ State Key Laboratory of Atmospheric Boundary Layer Physics and Atmospheric Chemistry, Institute of Atmospheric \\ Physics, Chinese Academy of Sciences, Beijing, China
}

(Manuscript received 30 September 2019, in final form 24 April 2020)

\begin{abstract}
The intermittency of atmospheric turbulence plays an important role in the understanding of particle dispersal in the atmospheric boundary layer and in the statistical simulation of high-frequency wind speed in various applications. There are two kinds of intermittency, namely, the magnitude intermittency (MI) related to non-Gaussianity and the less studied clusterization intermittency (CI) related to long-term correlation. In this paper, we use a $20 \mathrm{~Hz}$ ultrasonic dataset lasting for 1 month to study CI of turbulent velocity fluctuations at different scales. Basing on the analysis of return-time distribution of telegraphic approximation series, we propose to use the shape parameter of the Weibull distribution to measure CI. Observations of this parameter show that contrary to MI, CI tends to weaken as the scale increases. Besides, significant diurnal variations, showing that $\mathrm{CI}$ tends to strengthen during the daytime (under unstable conditions) and weaken during the nighttime (under stable conditions), are found at different observation heights. In the convective boundary layer, the mixed-layer similarity is found to scale the CI exponent better than the Monin-Obukhov similarity. At night, CI is found to vary less with height in the regime with large mean wind speeds than in the regime with small mean wind speeds, according to the hockey-stick theory.
\end{abstract}

\section{Introduction}

In the study of atmospheric turbulence, two kinds of intermittency are commonly discussed: the finescale intermittency (also called the inertial subrange intermittency, the internal intermittency, or the small-scale intermittency) and the global intermittency (also called the energy-containing intermittency) (Mahrt 1999). The scale of the global intermittency is normally from several minutes to hours, and the scale of the finescale intermittency is much smaller. For high-Reynolds-number atmospheric turbulence, the smallest scale of the inertial subrange is even much smaller than the resolution of the ultrasonic anemometer, which is commonly used to measure turbulent velocity in the atmospheric boundary layer (Kit et al. 2017). In this study, we focus on the finescale intermittency in the inertial subrange.

The finescale intermittency of turbulent velocity fluctuations was found to be prevalent in the atmospheric boundary layer (Schertzer et al. 1997; Boettcher et al. 2003; Vindel and Yagüe 2011; Wächter et al. 2012; Liu et al. 2019). To improve the modeling of turbulent flows (Friedrich and Peinke 1997; Baile et al. 2011; Calif and

Corresponding author: Fei Hu, hufei@mail.iap.ac.cn
Schmitt 2012) and obtain a better understanding of particle dispersal such as air pollutants and seeds (Wei et al. 2018; Duman et al. 2016) and the propagation of light and sound wave (Sreenivasan and Antonia 1997) in the atmospheric boundary layer, a better knowledge of the finescale intermittency of atmospheric turbulence is still needed.

The intermittency of turbulent velocity fluctuations at different spatial (time) scales can be exhibited by the statistics of velocity increments with different spatial (time) lags (Frisch 1995). For the atmospheric turbulence, because of widely used single-point measurements, the statistics of velocity increments with time lags is mostly studied. The probability density function of velocity increments is found to change from the nonGaussian long-tailed distribution at smaller lags to the Gaussian-like distribution at larger lags, which means that the turbulent velocity fluctuations are more likely intermittent at smaller scales (Castaing et al. 1990; Noullez et al. 2000; Boettcher et al. 2003; Liu et al. 2010; Liu and Hu 2013; Liu et al. 2019). Thus, the quantities quantifying deviation from the Gaussian distribution, such as the flatness (Frisch 1995; Tabeling et al. 1996; Bos et al. 2007), the average variation rate of cumulants (Malécot et al. 2000), and the Kullback-Leibler divergence 
(Granero-Belinchón et al. 2018), can be considered as measures of turbulence intermittency. Besides, the $p$ thorder moment of velocity increments, that is, the structure function, is generally found to scale with lag (Frisch 1995),

$$
\overline{|u(t+\tau)-u(t)|^{p}} \sim \tau^{\xi_{p}},
$$

where $u(t)$ is the velocity at time $t, \tau$ is the time lag, usually interpreted as the time scale of turbulence eddies in turbulence theories, and $\xi_{p}$, called the exponent of the structure function, is a function of $p$. According to the K41 theory of turbulence (Kolmogorov 1941), the exponent of the structure function $\xi_{p}$, is a linear function of $p$ for turbulence with the Gaussian distribution. Thus, the nonlinearity of $\xi_{p}$ is also widely used to measure turbulence intermittency (Schmitt et al. 1994; Lauren et al. 1999; Vindel and Yagüe 2011; Wei et al. 2017): the more nonlinear $\xi_{p}$ is, the more intermittent turbulence becomes.

In fact, except the intermittency related to nonGaussianity, there is another kind of intermittency related to clusterization. One can image that if extremes (defining by absolute values greater than a threshold) in a time series are moved together, the new series could be more intermittent than the original one, even if the series is Gaussian distributed. This kind of intermittency is called the clusterization intermittency, and the one related to non-Gaussianity is called the magnitude intermittency (Bershadskii et al. 2004). A famous example of clusterization intermittency is the fractional Brownian noise, which shows intermittency but is Gaussian distributed (Mandelbrot and Ness 1968; Ding and Yang 1995). Few studies have focused on the clusterization intermittency of turbulent velocity fluctuations in the atmospheric boundary layer. The analysis of vertical wind velocities shows that the boundary layer nonstationarity can affect statistics of clusterization intermittency ( $\mathrm{Li}$ and $\mathrm{Fu}$ 2013). It was found that the duration between clusters of nonstationary records is nearly power-law distributed, while the duration of stationary records is stretched exponential distributed. A series of systematic studies revealed that the clusterization intermittency is related to thermal stratification and roughness (Cava and Katul 2009; Cava et al. 2012). Recently, the interaction between clusterization intermittency and submesomotions in the stable boundary layer has gained much attention (Vercauteren and Klein 2015; Mortarini et al. 2018; Cava et al. 2019). Although above works found interesting results, many questions need further investigation. We want to know how the clusterization intermittency of atmospheric turbulence varies with turbulence scales, and how this kind of intermittency evolves with time, height, and surface-layer stability. The study could inform parameterization developments in the future study.
In this paper, we try to answer these questions by analyzing velocity increments with different time lags in the surface layer. There are two main differences between ours and previous studies. First, we use velocity increments, not wavelet analysis, proper orthogonal decomposition or other methods used in previous studies, to resolve turbulence scales. The use of velocity increments follows the convention in the study of magnitude intermittency. By using velocity increments, the difference between clusterization intermittency and magnitude intermittency with scale can be compared without considering systematic deviation among different scaleresolved methods, and the interpretation of clusterization intermittency by correcting or extending magnitude intermittency theories becomes possible. Second, our work stresses the connection between long-term statistical correlation and clusterization intermittency (see section 4), and basing on the connection, we propose a new measure to quantify clusterization intermittency (see section 5) and use it to analyze the characteristics of clusterization intermittency (see section 6).

\section{Data}

Data were collected in September 2009 at a site located in a steppe to the northeast of Xilinhaote $\left(44.124^{\circ} \mathrm{N}\right.$, $116.297^{\circ} \mathrm{E}$ ), in Inner Mongolia, China. The terrain, covered by grass about a few tens of centimeters high, is nearly horizontally uniform and flat, and the effect of heterogeneous terrain on clusterization intermittency is not considered in our analysis. Turbulence data, including high-frequency velocity and temperature, were collected by four ultrasonic anemometers (Campbell CSAT-3, $20 \mathrm{~Hz}$ ) deployed at 10-, 30-, 50-, and 70-m levels on a $100-\mathrm{m}$ tower. The details about the experiments are available in Lyu et al. (2018).

The quality control algorithms, proposed by Vickers and Mahrt (1997), are used to find spikes, data with the amplitude resolution problem, dropouts, data violating absolute limits, data with unphysical high-order moments, and discontinuities. Records with unphysical high-order moments or discontinuities are discarded in the analysis. If the percentage of empty values or data violating absolute limits is greater than $0.5 \%$, the record is not used in the analysis. If the percentage of spikes is greater than $1 \%$, if the percentage of data with the amplitude resolution problem is greater than $70 \%$, or if the dropouts near mean and extreme are greater than $6 \%$ and $10 \%$, respectively, the record is also not used in the analysis. Besides, records seriously contaminated by white noises in the high-frequency range are also discarded. After the quality control, about $93.2 \%$ of $10-\mathrm{m}$, $89.7 \%$ of $30-\mathrm{m}, 93.0 \%$ of $50-\mathrm{m}$, and $89.5 \%$ of $70-\mathrm{m}$ 


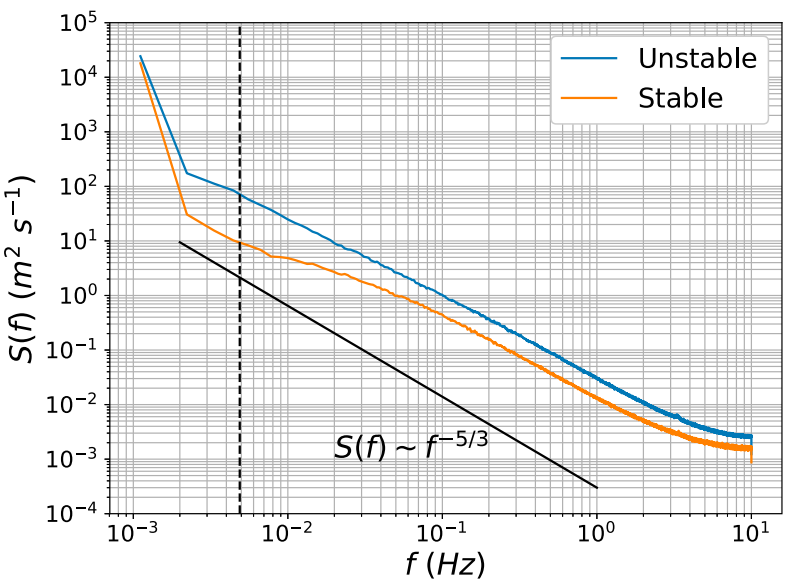

FIG. 1. Wind speed spectra in the stable and unstable situations. The vertical broken line marks the frequency of $1 / \tau_{\max }$, where $\tau_{\max }$ (204.8 s) is the largest time lag of wind speed increments in this study.

records remain. The problematic data in the remaining records are deleted and interpolated by the cubic spline method. The fraction of problematic data is $0.06 \%$, $0.14 \%$, and $0.13 \%$ for horizontal velocity, vertical velocity, and temperature, respectively.

To compute the Obukhov length and the friction velocity, we transform the instrument reference frame to the streamline reference by using double rotation (Kaimal and Finnigan 1994). In this paper, we mainly analyze the wind speed, that is,

$$
u=\sqrt{u_{1}^{2}+u_{2}^{2}+u_{3}^{2}},
$$

where $u_{1}, u_{2}$, and $u_{3}$ are velocity components in the longitudinal, lateral, and vertical directions, respectively. Records are chronologically grouped into many samples each lasting $15 \mathrm{~min}$, and the average period is also set to $15 \mathrm{~min}$, unless otherwise noted. The double rotation of reference frame and the analysis method in sections 4 and 5 is applied on each 15-min sample. The choice of 15-min averaging time is based on the analysis of Bou-Zeid et al. (2010), where the sensitivity of turbulence statistics to averaging time is tested and no significant effect on results with averaging time from $15 \mathrm{~min}$ to $1 \mathrm{~h}$ is found.

The wind speed increments are used to resolve turbulence scales. The time lag of wind speed increment, representing characteristic time scale of turbulent eddies, is from 0.05 to $204.8 \mathrm{~s}$ in this study. The averaged spectra in the unstable and stable situations are shown in Fig. 1. The spectrum in the inertial subrange is approximately proportional to $f^{-5 / 3}$ (Kolmogorov 1941). The frequency of $1 / \tau_{\max }$ is marked by a broken vertical line in Fig. 1, where $\tau_{\max }=204.8 \mathrm{~s}$ is the largest time lag. It shows that the time scales in this study are mainly in the inertial subrange.
The deviation from $f^{-5 / 3}$ between $1 / \tau_{\max }$ and about $1 / 50 \mathrm{~Hz}$ in the stable spectrum would be related to the contamination of submesomotions. The results calculated with the largest time lag, $50 \mathrm{~s}$ (not shown), have been compared with those with $204.8 \mathrm{~s}$, and no significant difference is found. Thus, the choice of $\tau_{\max }=204.8 \mathrm{~s}$ does not significantly impact the conclusions in the stable situations.

\section{Interpretation of clusterization intermittency}

In this section, we use time series models to interpret clusterization intermittency. Figure 2 shows the time series models of magnitude and clusterization intermittency, and their comparisons with wind speed time series. The details about these models are referred to Mantegna and Stanley (1999). The Lévy flight is a model of magnitude intermittency. A series of the Lévy flight with Cauchy distributed increments is shown in Fig. 2a and the adjacent increments of this series are shown in Fig. 2b. The non-Gaussian heavy-tailed distribution of increments makes large values more likely to occur than Gaussian white noises (see the red line in Fig. 2d). The Lévy flight increment series thus seems data with spikes (Fig. 2b). The fractional Brownian motion is a model of clusterization intermittency. A series of the fractional Brownian motion with a Hurst exponent of 0.9 is shown in Fig. $2 \mathrm{c}$ and the adjacent increments of this series are shown in Fig. 2d. Although the fractional Brownian increments are Gaussian distributed, they seem to be more intermittent than the Gaussian white noises because large values in the former tend to appear in clusters.

To illustrate the analysis, we choose two 15-min samples respectively observed in the daytime unstable boundary layer and the nocturnal stable boundary layer during the same day. The two samples are labeled by case A (unstable, see Fig. 2e) and case B (stable, see Fig. 2g); their flow characteristics are listed in Table 1. Similar to the fractional Brownian motion increments, large values of wind speed increments tend to appear in clusters, especially for the case in the unstable boundary layer (see Figs. 2f,h). In fact, atmospheric turbulence is a mixture of clusterization intermittency and magnitude intermittency. The magnitude intermittency has been extensively discussed. In this paper, we mainly focus on clusterization intermittency and propose a method to extract and quantify clusterization intermittency.

\section{Long-term correlation and clusterization intermittency}

From the view of the random process, Bunde et al. (2005) found that the clusterization intermittency is 

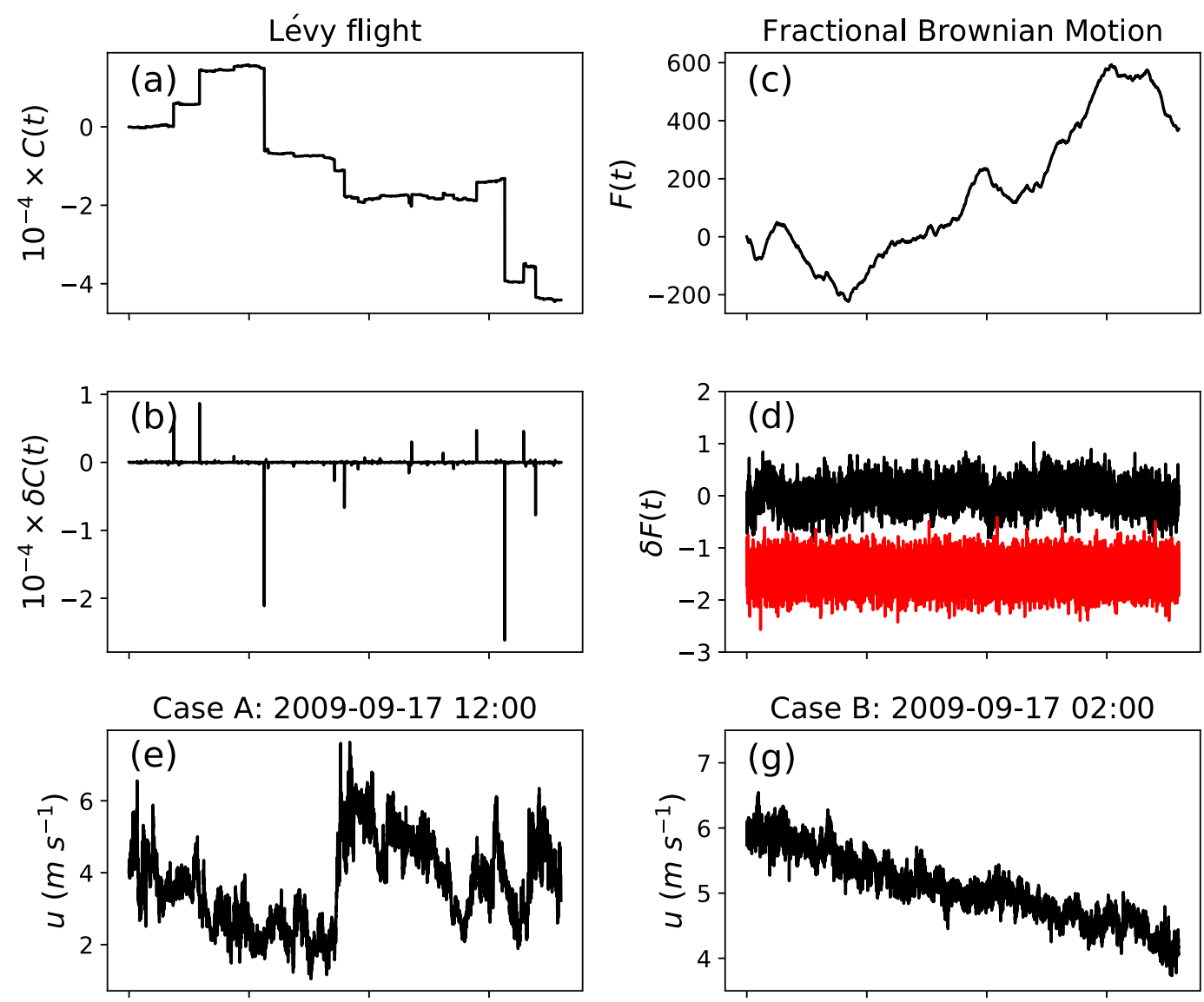

Case B: 2009-09-17 02:00
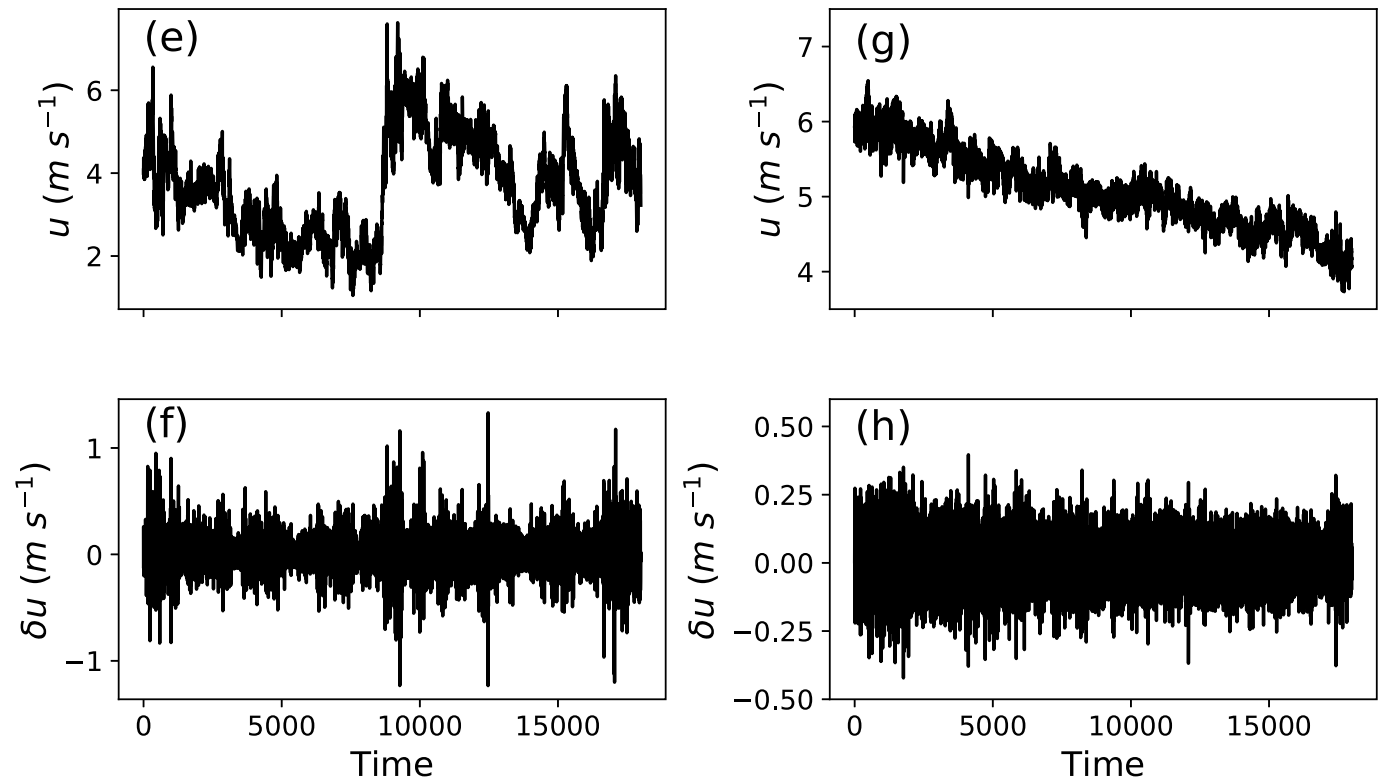

FIG. 2. The time series of (a) the Lévy flight $C(t)$ and (c) the fractional Brownian motion $F(t)$. The increments of (b) $C(t)$ and (d) $F(t)$ between adjacent samples. The increments of the Lévy flight are Cauchy distributed with the shape parameter of 1 . The fractional Brownian motion has the Hurst exponent of 0.9. In (d) the Gaussian white noises (red line) have the same mean and standard derivation as the fractional Brownian motion increments. The 15-min time series of (e),(g) wind speeds $u$ and (f),(h) the adjacent increments $\delta u$ for (e),(f) case A and $(\mathrm{g}),(\mathrm{h})$ case B.

intimately related to the long-term correlation in many numerical and natural signals. The long-term correlation means that the autocorrelation function, measuring correlation between two different times in a time series, decreases much slower than the exponential-like dropoff. In this section, we analyze the autocorrelation function of turbulent wind speed fluctuations at different scales and discuss the relationship between the long-term correlation and clusterization intermittency.

\section{a. Autocorrelation function}

The multifractal random walk (MRW), proposed by Bacry et al. (2001), is widely used to describe the time series with small-scale multiscaling, non-Gaussianity, 
TABLE 1. Flow characteristics of two samples shown in Figs. 2e and $2 \mathrm{~g}$. The surface-layer stability is measured by $z / L$, where $z$ is the observation height and $L$ is the Obukhov length.

\begin{tabular}{lcc}
\hline \hline & Case A & Case B \\
\hline Mean wind speed $\left(\mathrm{m} \mathrm{s}^{-1}\right)$ & 3.68 & 5.08 \\
Mean temperature $\left({ }^{\circ} \mathrm{C}\right)$ & 14.66 & 11.27 \\
Friction velocity $\left(\mathrm{m} \mathrm{s}^{-1}\right)$ & 0.18 & 0.03 \\
Stability $z / L$ & -14.02 & 12.50 \\
Observation height $(\mathrm{m})$ & 30 & 30 \\
Observation time $(\mathrm{LST})$ & 1200 & 0200 \\
\hline
\end{tabular}

intermittency, and long-term correlation. Recently, the turbulent velocity fluctuations in the atmospheric boundary layer were found to be well described by the MRW generated by a lognormal cascade process (hereafter the lognormal MRW) (Liu et al. 2019). We use this model to discuss the autocorrelation function of turbulent velocity fluctuations. The details about the universal MRW theory are referred to Muzy and Bacry (2002) and Bacry and Muzy (2003).

Assuming that the velocity increments follow a lognormal MWR with a Hurst exponent of 0.5 , the $p$ thorder autocorrelation function of absolute increments at scale $\tau$ is

$$
C_{p}(t, \tau)=\overline{\left|\delta u_{\tau}(t)\right|^{p}\left|\delta u_{\tau}(0)\right|^{p}} \sim\left(\frac{\tau}{T}\right)^{2 \xi_{p}}\left(\frac{t}{T}\right)^{-\mu p^{2} / 4},
$$

which holds when $\tau \ll t \leq T$ and $\mu>0$ (Bacry et al. 2001). The symbol $\delta u_{\tau}(t)$ denotes the velocity increment between time $t+\tau$ and $t$, and its $p$ th-order absolute moments scale with $\tau$ :

$$
\overline{\left|\delta u_{\tau}\right|^{p}} \sim \tau^{\xi_{p}}
$$

where $\xi_{p}$ is the exponent of structure function with a Hurst exponent of 0.5 , that is,

$$
\xi_{p}=\frac{p}{2}\left(1+\frac{\mu}{2}\right)-\frac{\mu p^{2}}{8} .
$$

The parameter $\mu$ in Eq. (3) is the magnitude intermittency exponent, which defines whether the increments are Gaussian $(\mu=0)$ or non-Gaussian $(\mu>0)$.

Generally, Liu et al. (2019) have verified the Hurst exponent in the lognormal MRW is apparently less than 0.5 for turbulent wind speeds in the atmospheric boundary layer. Thus, Eq. (3) cannot be used to describe the autocorrelation properties of turbulent wind speed data. Mathematically, it is difficult to derive the $p$ th autocorrelation function of the lognormal MRW with an arbitrary Hurst exponent. If we replace the structure function exponent $\xi_{p}$ with the Hurst exponent $H=1 / 2$ in Eq. (3) by an exponent $\xi_{p, H}$ with an arbitrary Hurst exponent $H$, and replace the magnitude intermittency exponent $\mu$ by an arbitrary parameter $\gamma$, an assumption of autocorrelation function of turbulent wind speeds is obtained:

$$
C_{p}(t, \tau) \sim\left(\frac{\tau}{T}\right)^{2 \xi_{p, H}}\left(\frac{t}{T}\right)^{-\gamma p^{2}},
$$

where $\tau \ll t \leq T, \gamma>0$, and the Hurst exponent $H \in(0$, $1)$. It has been strictly proved that

$$
\xi_{p, H}=p H(1-m)-\mu \frac{p^{2} H^{2}}{2},
$$

with an arbitrary parameter $m \in R$ (Muzy and Bacry 2002; Bacry and Muzy 2003). The direct verification of Eq. (6) is not easy. Due to limited data, the autocorrelation function would be scattered at large $t$, where Eq. (6) is assumed to hold. We thus verify it indirectly. According to Eq. (6),

$$
G_{p}(t) \equiv \frac{C_{p}(t, \tau)}{(\tau / T)^{2 \xi_{p, H}}} \sim\left(\frac{t}{T}\right)^{-\gamma p^{2}} .
$$

Then, the function $G_{p}(t)$ is independent of $\tau$, and

$$
G_{p} \sim\left(\frac{t}{T}\right)^{-\gamma(p-1)^{2}[p /(p-1)]^{2}} \sim G_{p-1}^{\zeta_{p}},
$$

where

$$
\zeta_{p}=\left(\frac{p}{p-1}\right)^{2}
$$

The verification of Eq. (6) is thus replaced by the verification of Eq. (9).

Figure 3 shows the verification of Eq. (9) for case A and case B. We compute $C_{p}(t, \tau)$ at scales $\tau=0.05,10$, 50 , and $100 \mathrm{~s}$ and find that for a fixed $p$, if $C_{p}(t, \tau)$ is divided by $(\tau / T)^{2 \xi_{p}, H}$, all data will collapse into a single curve in the plot of $G_{p}$ versus $G_{p-1}$. We then fit the data at $\tau=0.05 \mathrm{~s}$ with a linear function $\log G_{p}=\zeta_{p} \log G_{p-1}+$ $B$ in the $\log -\log$ plot, where $B$ is the only fitting parameter, and find that Eq. (10) shows a good fit to the data. We have verified all samples and found that $G_{p}$ is independent of scale $\tau$ for the most samples, and more than $75 \%$ coefficients of determination are greater than 0.9 for $p=2,3, \ldots, 10$, as shown in Fig. 3. Thus, Eq. (9) is true for most samples, and Eq. (6) is indeed a reasonable assumption for the autocorrelation function of turbulent velocity fluctuations in the atmospheric boundary layer. The power exponent of autocorrelation function in Eq. (6) reflects how slowly correlation decays with time in a time series. In the next section, the analysis of this exponent reveals that long-term correlations exist in the time series of wind speed increments and is related to 

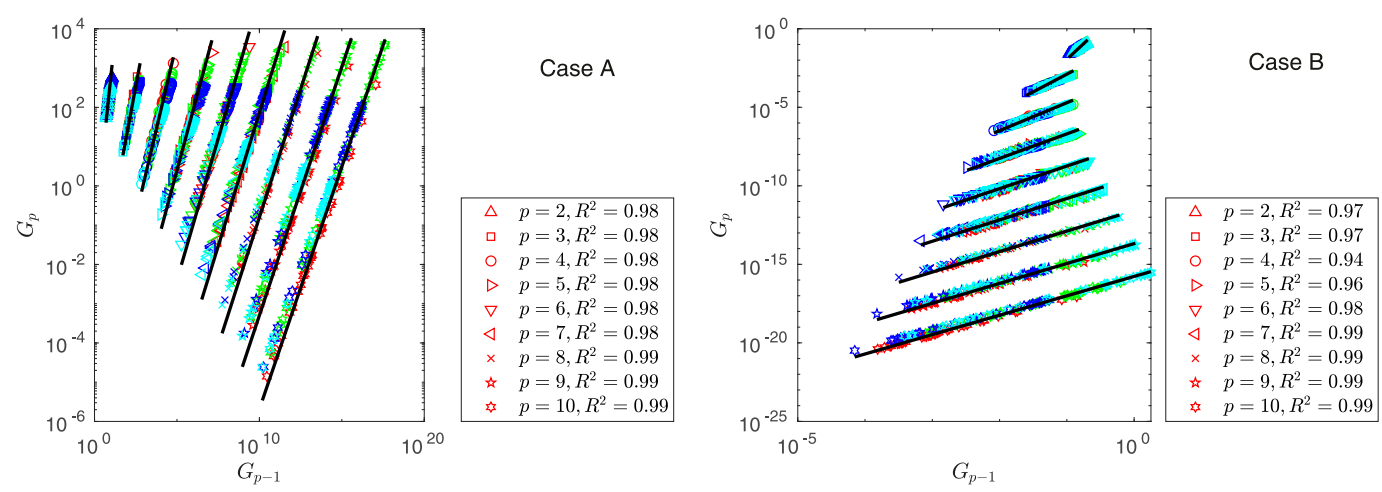

FIG. 3. Verification of Eq. (9) for (left) case A and (right) case B. The function $G_{p}$ is computed at scale $\tau=0.05$ (red), 10 (green), 50 (blue), and $100 \mathrm{~s}$ (cyan) for different values of order $p$ (denoted by different symbols in the legends). Data at scale $\tau=0.05 \mathrm{~s}$ are fitted to Eq. (10), and the corresponding coefficients of determination $R^{2}$ are shown in the legends. For clarity, the $y$-axis values are multiplied by a factor of $10^{-2(p-2)}$

clusterization intermittency $(\mathrm{CI})$, which inspires us to propose a method to quantify clusterization intermittency in section 5 .

\section{b. Fluctuation analysis}

In this section, we discuss the parameter $\gamma$ in Eq. (6). The first-order autocorrelation function $C_{1}(t, \tau)$ shows the correlations in the time series of absolute increments. We have shown that $C_{1}(t, \tau) \sim t^{-\gamma}$ in section $4 \mathrm{a}$, where the parameter $\gamma$ is independent of scale $\tau$. The parameter $\gamma$ is called the correlation exponent, because it defines the correlation type. If $\gamma$ lies in $(0,1)$, $\int_{0}^{\infty} C_{1}(t, \tau) d t=\infty$, and the time series of absolute increments are thus long-term correlated (Beran 1994), while for the independent or short-term correlated absolute values, $\int_{0}^{\infty} C_{1}(t, \tau) d t<\infty$. Because of limited data, the autocorrelation function is usually scattered at large $t$. Thus, it is difficult to verify whether $\gamma$ lies in $(0,1)$ by analyzing autocorrelation function. Many methods, although controversy exists, have been proposed to estimate $\gamma$ (Beran 1994; Taqqu et al. 1995; Franzke et al. 2012). The fluctuation analysis, proposed by Peng et al. (1992), is the one widely used in the literature.

The fluctuation analysis is described as follows. The profile of a discrete time series $x_{i}(i=1,2, \ldots, N)$ is defined by

$$
Y_{n}=\sum_{i=1}^{n} x_{i}
$$

where $n=1,2, \ldots, N$. Then, the profile increment with lag $s$ is obtained by $\delta Y_{s}=Y_{n+s}-Y_{n}$. If the standard deviation of $\delta Y_{s}$, called the fluctuation function, scales with large values of $s$, that is,

$$
F(s) \equiv\left(\overline{\delta Y^{2}}-\overline{\delta Y^{2}}\right)^{1 / 2} \sim s^{\alpha},
$$

where $\alpha$ is called the fluctuation exponent, one can prove that (Kantelhardt et al. 2001)

$$
\alpha=1-\frac{\gamma}{2},
$$

where $\gamma$ is the correlation exponent of the time series $x_{i}$. Equation (13) shows that if the time series is long-term correlated, $\gamma \in(0,1)$, and thus $\alpha \in(0.5,1)$. If the time series is independent, $\alpha=0.5$.

The fluctuation analysis is used to analyze the absolute velocity increments of case $A$ and case $B$ (see circles in Figs. 4a,b). Because the correlation exponent is independent of scale $\tau$, we just choose $\tau=0.05 \mathrm{~s}$ in the analysis. Results show that the fluctuation exponent is significantly greater than 0.5 but less than 1 for each case. Similar results are also found for most of the other samples (see "raw" in Fig. 4c). We then randomly shuffle the data and perform the same fluctuation analysis for the shuffled data. The randomly shuffling removes clusterization intermittency from data, but does not affect the underlying distribution and thus the magnitude intermittency. For case A and case B, the fluctuation exponents of the shuffled data are found to be approximately 0.5 (see squares in Figs. 4a,b). The analysis has been done for the whole samples, and we find that the fluctuation exponents of shuffled data are also around 0.5 , similar to the fluctuation exponents of independent Gaussian samples (see Fig. 4c). We thus conclude that the absolute velocity increments in the atmospheric boundary layer are generally long-term correlated, and the clusterization intermittency is intimately related to the long-term correlation. If the clusterization intermittency disappears, the correlation in the time series changes from the longterm type to the short-term or the independent type.

The magnitude intermittency exponent $\mu$, obtained by fitting data to Eq. (7), is plotted versus the correlation 

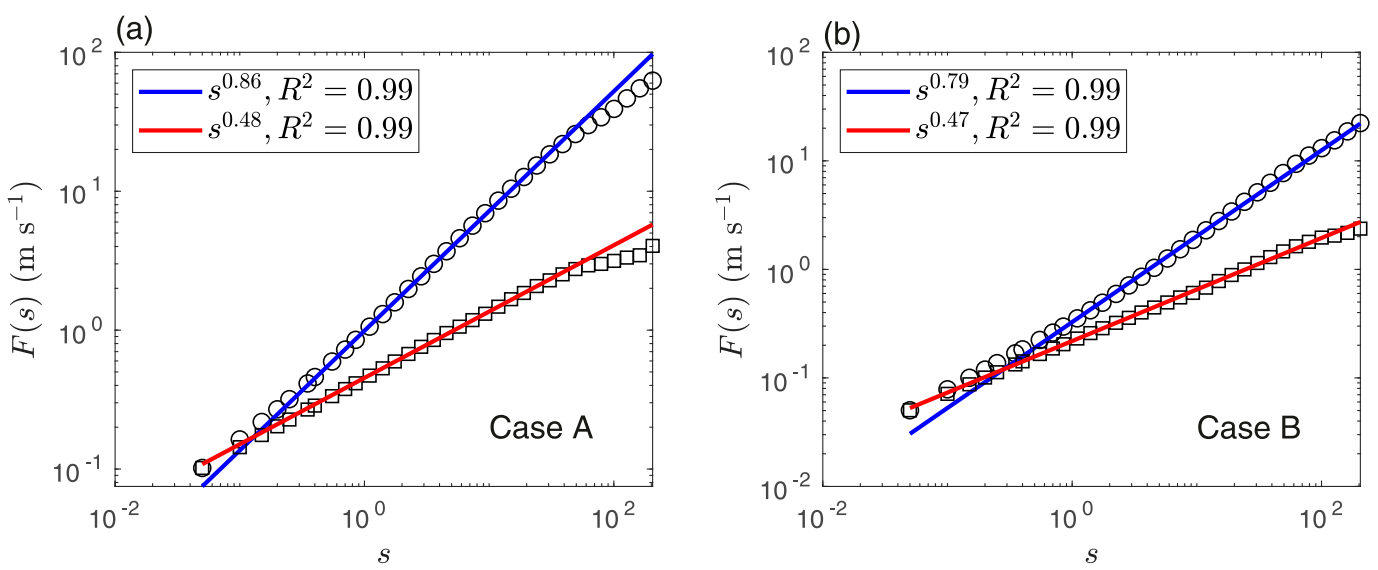

(c)
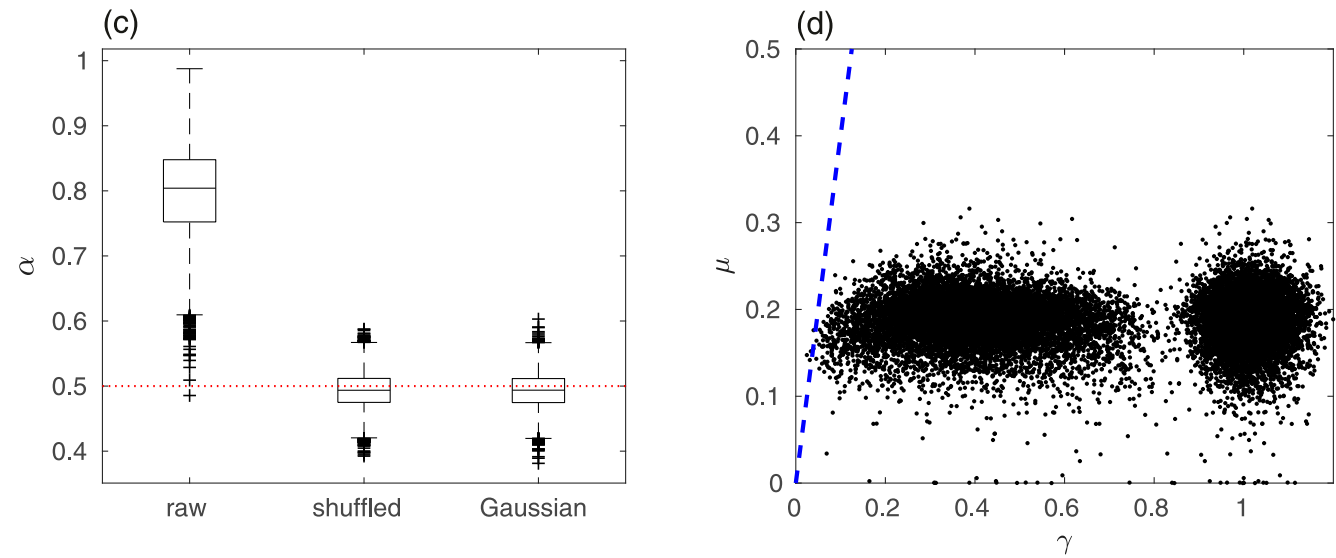

FIG. 4. The fluctuation analysis of absolute velocity increments at scale $\tau=0.05 \mathrm{~s}$ for (a) case A and (b) case B. The fluctuation functions $F(s)$ of raw data and the corresponding randomly shuffled data are shown by circles and squares, respectively. (c) A boxplot of the fluctuation exponents $\alpha$ for all raw samples, compared with the corresponding randomly shuffled samples and the independent Gaussian samples (with mean $=0$ and standard deviation $=1$ ) with the same size of the raw samples. (d) The magnitude intermittency exponent $\mu$ vs the correlation exponent $\gamma$, estimated by the fluctuation exponent [see Eq. (13)]. The broken line shows the prediction of Eq. (3), that is, $\mu=4 \gamma$.

exponent $\gamma$, obtained by the fluctuation analysis. Results show that deviating from the prediction of Eq. (3), $\gamma$ is almost independent of $\mu$ (see Fig. 4d). This indicates that the clusterization intermittency is unrelated to the magnitude intermittency. They are two kinds of intermittency, not just two facets of the same intermittency. It means that clusterization and magnitude intermittency could be caused by two kinds of physical mechanisms. Distinguishing clusterization and magnitude intermittency is a first step to understand the underlying intermittency mechanisms.

\section{Clusterization intermittency exponent}

In this section, we discuss the clusterization intermittency exponent that can measure this kind of intermittency. The zero-crossing statistics of the telegraphic approximation of time series is commonly used to design clusterization intermittency exponent (Bershadskii et al. 2004; Sreenivasan and Bershadskii 2006; Cava et al. 2012;
$\mathrm{Li}$ and $\mathrm{Fu}$ 2013; Cava et al. 2019). We briefly review this method and then discuss its flaws (section 5b). Basing on above analysis of long-term correlation, we propose a new, better exponent (section 5c).

\section{a. Telegraphic approximation}

The magnitude and clusterization intermittency always coexist in a time series. Before defining measures, one should separate the two kinds of intermittency. This can be simply done by the telegraphic approximation (TA) of a time series $x(t)$ (Bershadskii et al. 2004):

$$
\operatorname{TA}(x)=\frac{1}{2}\left[\frac{x(t)-h}{|x(t)-h|}+1\right] .
$$

By definition, the TA transforms the values of $x(t)$ greater than $h$ to 1 and the others to 0 (see Fig. 5). In practice, the threshold $h$ should be set carefully. If $h$ is set to be very large, the number of events greater than 

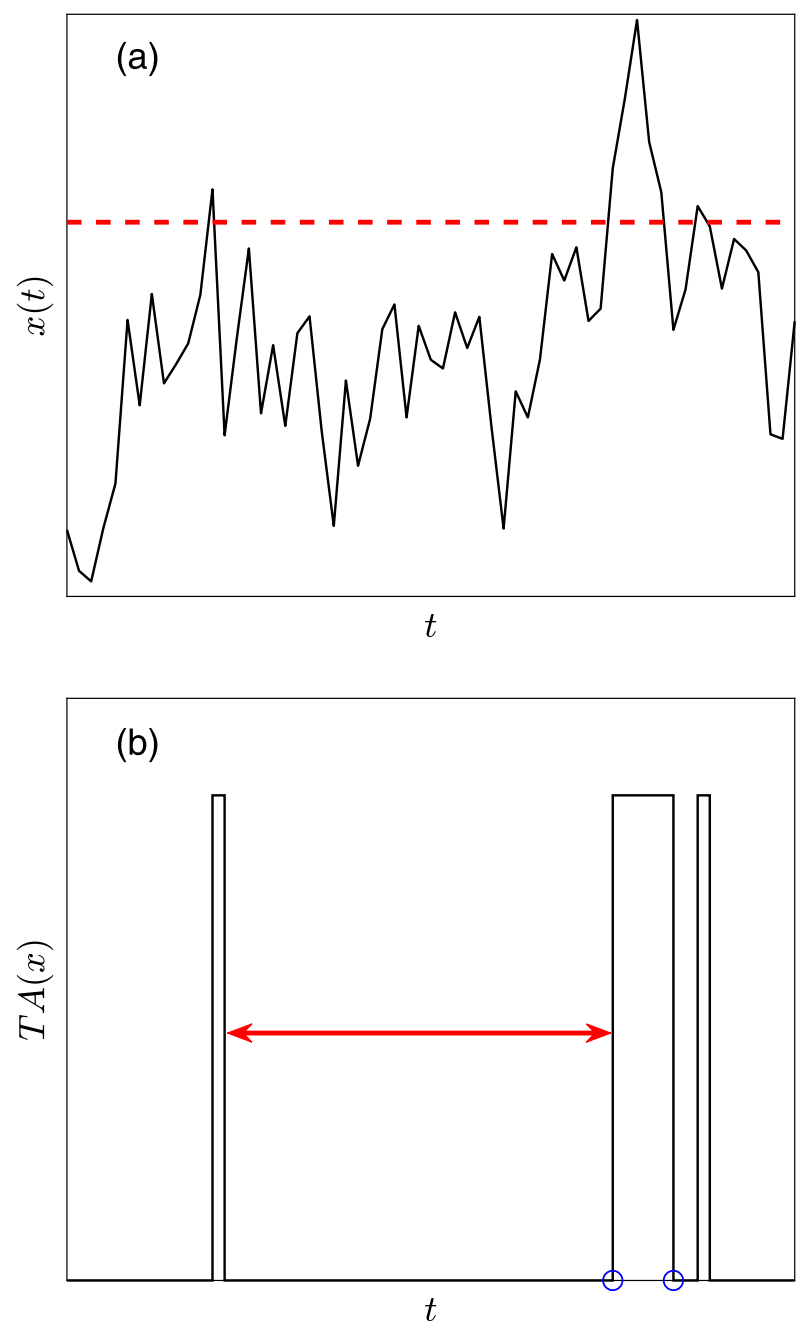

FIG. 5. Illustration of telegraphic approximation TA $(x)$ of a time series $x(t)$. (a) The broken line in shows the threshold. (b) The double arrows in mark the maximum return time between telegraphic approximation events. The circles in (b) denote the zero crossings of the longest telegraphic approximation event.

the threshold are very small and $\operatorname{TA}(x)$ is sparse; if $h$ is set to be very small, $\operatorname{TA}(x)$ is occupied by a few long events. To avoid poor statistics in both cases, the threshold should be set to some intermediate value. The choice of threshold is further discussed in section $5 \mathrm{c}$.

\section{b. Zero-crossing density}

The zero crossing is a time point where TA $(x)$ switches from 1 to 0 or from 0 to 1 (see Fig. 5). The zero-crossing density $n_{l}$ is computed by counting the number of zero crossings in a running time window of size $l$ and dividing this number by the total number of data in the window. The standard deviation of zero-crossing density $n_{l}$ is usually assumed to be a power-law function of window size $l$; that is,
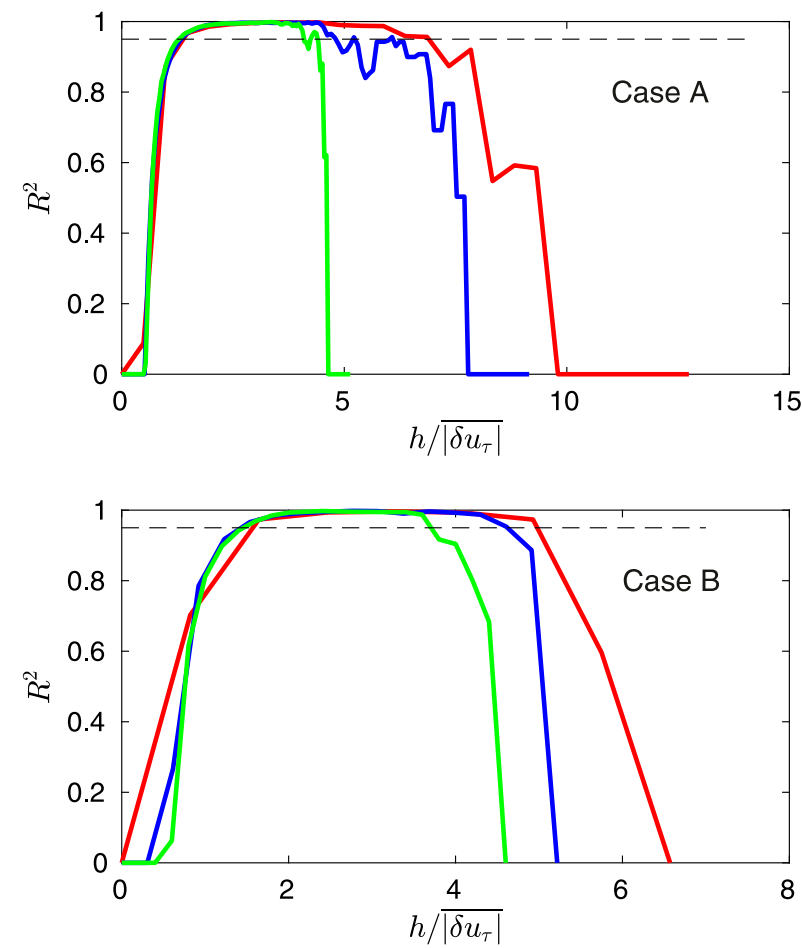

FIG. 6. Threshold analysis for (top) case A and (left) case B. The return-time distribution of randomly shuffled absolute velocity increments is fitted to the exponential distribution, and the coefficients of determination $R^{2}$ at scale $\tau=0.05$ (red line), 10 (blue line), and $100 \mathrm{~s}$ (green line) are plotted as a function of dimensionless threshold, where $\overline{\left|\delta u_{\tau}\right|}$ is the mean absolute velocity increment. The broken lines denote $R^{2}=0.95$.

$$
\left(\overline{n_{l}^{2}}-\mu_{l}^{2}\right)^{1 / 2} \sim l^{-d},
$$

where $\mu_{l}$ is the mean of $n_{l}$ and $d$ is a measure of clusterization intermittency. The smaller than $1 / 2$ the $d$ is, the more clusterization intermittency becomes.

There are some flaws in the use of zero-crossing density to measure clusterization intermittency. First, in this method, $d$ is assumed to equal $1 / 2$ for any time series without clusterization intermittency. This assumption may be not generally true. The mathematical derivation of the zero-crossing statistics is very difficult. As far as we known, $d$ is strictly proved to equal $1 / 2$ just for the stationary Gaussian process with weak correlation (Kratz 2006), taken as an example without clusterization intermittency. Whether this conclusion is true for the nonGaussian process is still an open problem. Because velocity increments at small scales are generally non-Gaussian, it seems unreasonable to measure clusterization intermittency by qualifying deviation of $d$ from $1 / 2$. Second, for the Gaussian process with long-range dependence, it has been proved that Eq. (15) is indeed true for very large window size (Slud 1994). However, it also indicates that it is difficult 

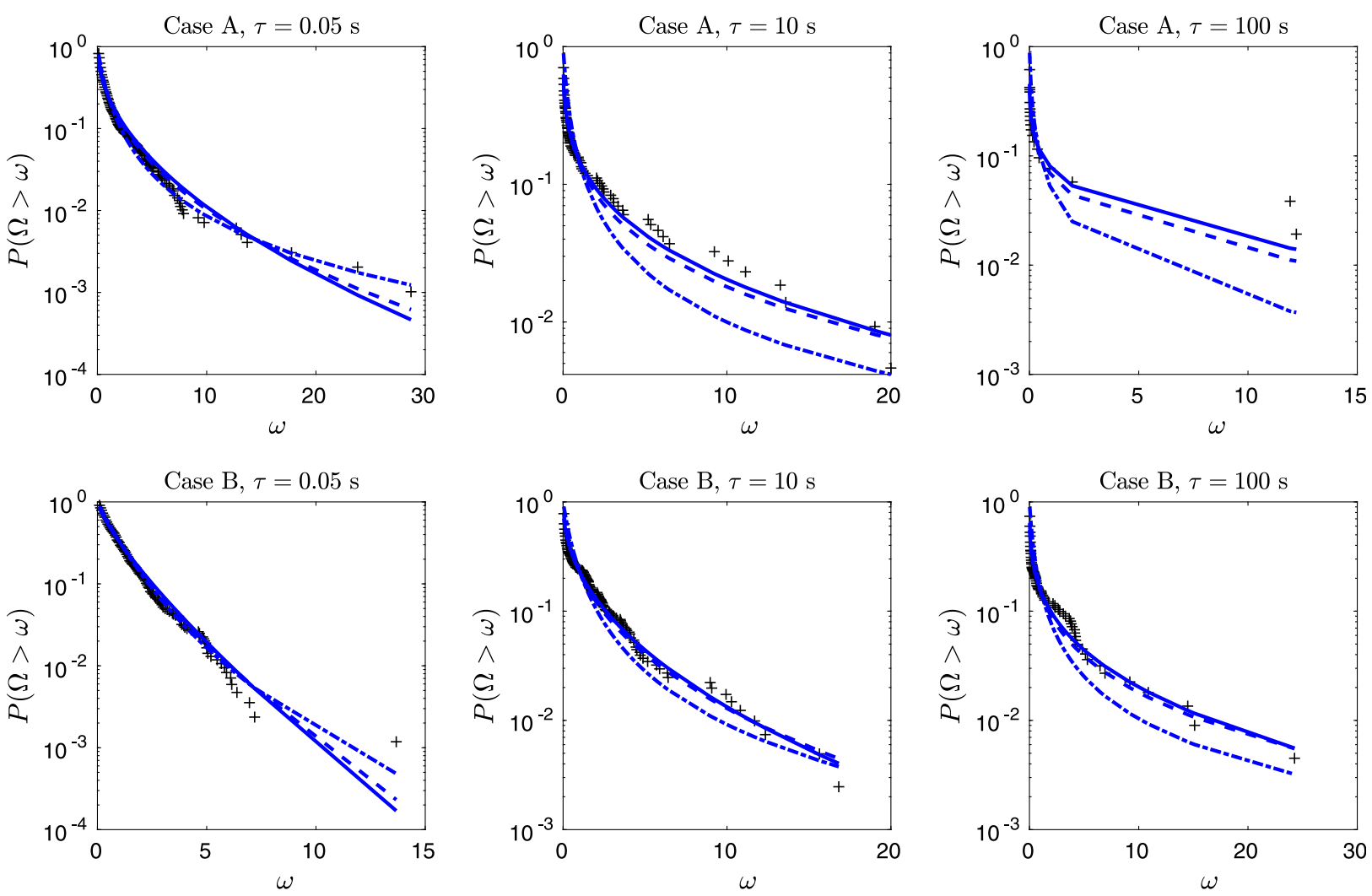

FIG. 7. Return-time tail distributions of telegraphic approximation of absolute velocity increments, $P(\Omega>\omega)$, at scale $\tau=($ left $) 0.05$, (center) 10, and (right) $100 \mathrm{~s}$ for (top) case A and (bottom) case B (points). Data are fitted to the Weibull distribution (line), the stretched exponential distribution (broken line), and the $q$-exponential distribution (dash-dotted line).

obtain an accurate estimate of $d$, because the statistics is poor with large window size. Therefore, we propose a better measure, namely the return-time distribution, to quantify clusterization intermittency.

\section{c. Return-time distribution}

The return time, also called the waiting time or the interpulse period, is the duration between events with values greater than a threshold (see Fig. 5). For the statistically independent time series, the return time is strictly proved to be exponential distributed (Karlin and Taylor 1975; Liu et al. 2014):

$$
f(\Omega)=\exp (-\Omega),
$$

where $\Omega$ is the normalized return time, and $f(\Omega)$ is the probability density function of $\Omega$. The normalized return time $\Omega$ is defined by $r / \bar{r}$, where $r$ is the return time, and $\bar{r}$ is its mean. Unlike the zero-crossing statistics, the exponential distribution is true for any statistically independent random process, whether it is Gaussian or non-Gaussian. Thus, the exponential distribution can be reasonably considered as a benchmark to measure clusterization intermittency.
We here return to the problem of threshold choice. For a randomly shuffled time series, having no correlations and clusterization intermittency (see section $5 b$ ), the return-time distribution must be the exponential distribution. However, an unsuitable choice of threshold could cause poor statistics, and thus, the exponential distribution would not be observed, even for the

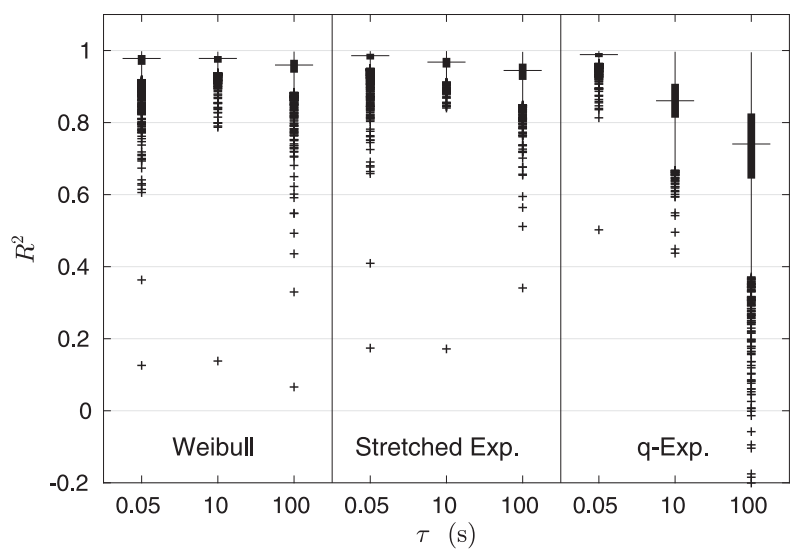

FIG. 8. A boxplot of coefficients of determination $R^{2}$ between models and the measured distributions of all samples. 

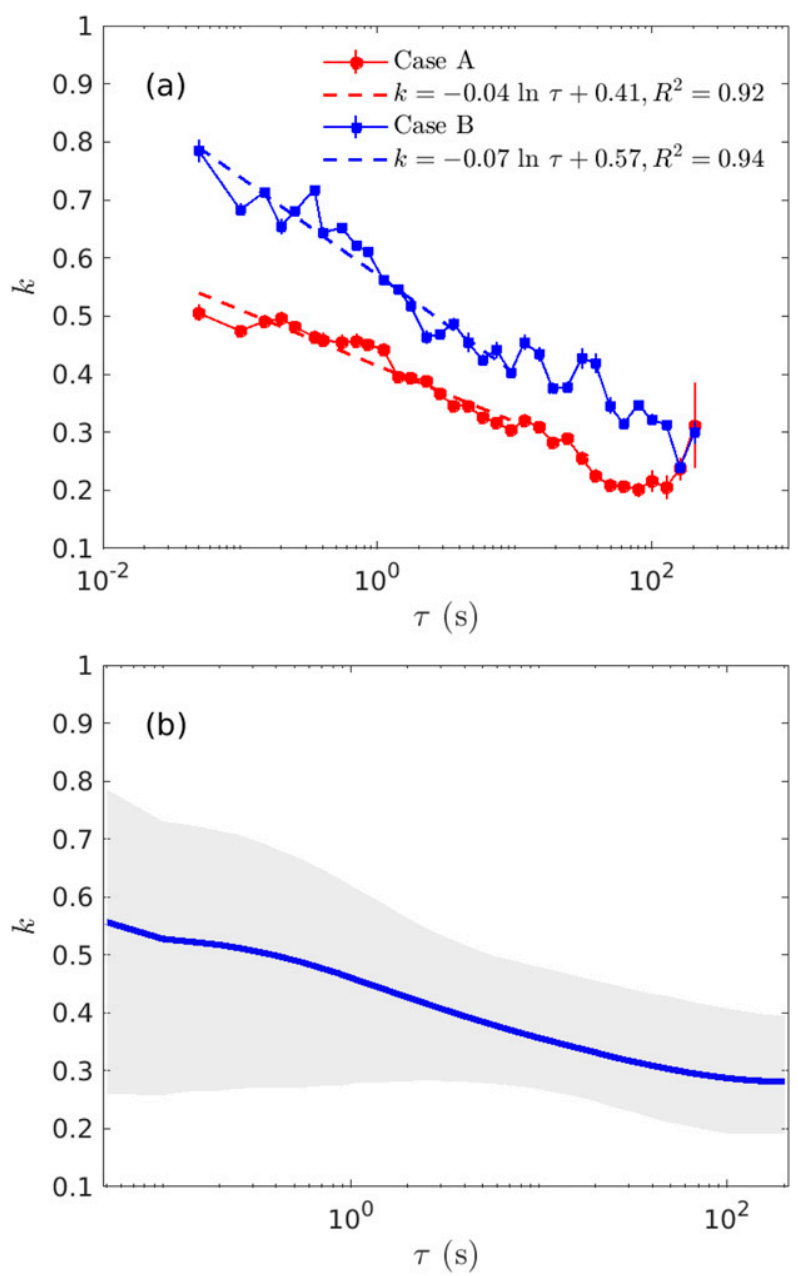

FIG. 9. The clusterization intermittency exponent $k$ vs scale $\tau$ for (a) case A (red circles) and case B (blue squares) and (b) all samples. The gray area in (b) shows the range of observed $k$, and the solid line shows the sample average.

independent time series. We randomly shuffle the absolute wind speed time series, choose a threshold, and make the telegraphic approximation of this series, and then calculate its return-time distribution. If the measured distribution can be well fitted by the exponential distribution, the threshold is considered to be suitable. The procedure of threshold choice is illustrated in Fig. 6 , where the coefficients of determination $R^{2}$ between the measured distribution and the exponential distribution at different scales are shown. As discussed in section 5a, $R^{2}$ declines for both very small and very large thresholds. Because sample size decreases with increasing scale, $R^{2}$ declines earlier at larger scales when the threshold is very large. A suitable threshold can be chosen in the intermediate plateau range, where $R^{2}>$ 0.95 . For case A, the threshold can be set between 1.5 and 4 times of mean absolute velocity increment for all

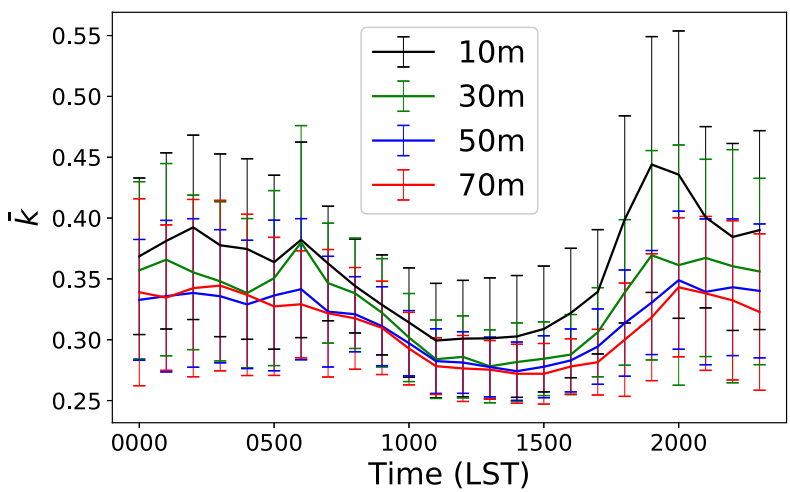

FIG. 10. Diurnal variation of scale-averaged clusterization intermittency exponent $\bar{k}$ at different observation heights. The standard deviation of $\bar{k}$ is denoted by an error bar.

scales. For case B, the threshold can be set between 2 and 3 times of mean absolute velocity increment for all scales. We have analyzed all samples and find that if the threshold is set between 2 and 3 times of mean absolute velocity increment for all scales, more than $90 \%$ samples have $R^{2}>0.95$. In the following analysis, the threshold is set to 2.5 times of mean absolute velocity increments. With this threshold, about $95.8 \%$ samples have $R^{2}>0.95$.

Whether there is a universal and analytical return-time distribution for the long-term correlated time series is still an open problem. Models have been proposed in various applications. Here, we introduce some commonly used models. All of them include the exponential distribution as a specific case for the statistically independent time series.

(i) The stretched exponential distribution (Bunde et al. 2005; Altmann and Kantz 2005):

$$
f(\Omega)=a_{\kappa} \exp \left[-\left(b_{\kappa} \Omega\right)^{\kappa}\right],
$$

where

$$
a_{\kappa}=b_{\kappa} \frac{\kappa}{\Gamma(1 / \kappa)}, \quad b_{\kappa}=\frac{2^{2 / \kappa} \Gamma\left(\frac{2+\kappa}{2 \kappa}\right)}{2 \sqrt{\pi}},
$$

for $0<\kappa \leq 1$. The tail distribution of the stretched exponential distribution is

$$
P(\Omega>\omega)=\int_{\omega}^{\infty} f(\Omega) d \Omega=\frac{a_{\kappa}}{b_{\kappa} \kappa} \Gamma\left[\frac{1}{\kappa},\left(\omega b_{\kappa}\right)^{\kappa}\right] .
$$

If $\kappa=1$, the stretched exponential distribution is just the exponential distribution for the statistically independent time series. By numerical analysis, Eichner et al. (2007) found that the stretched exponential distribution can only fit large return time, while small return time is well fitted by the power-law distribution. A similar result is also found for atmospheric 


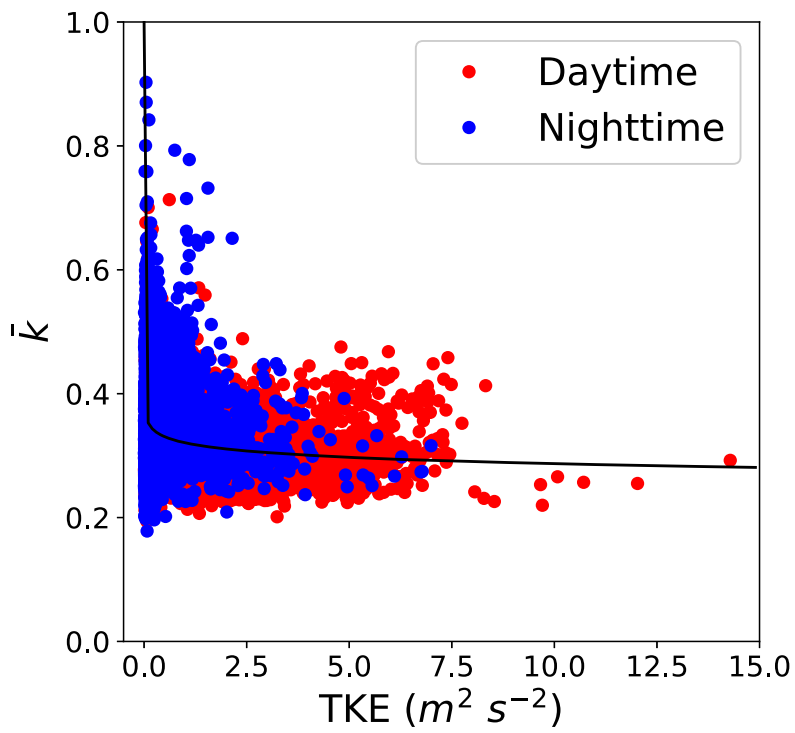

FIG. 11. Dependence of scale-averaged clusterization intermittency exponent $\bar{k}$ with turbulence kinetic energy (TKE) during daytime (red dots) and nighttime (blue dots). The line shows a least squares fitting function of $\bar{k}=1-\left(0.80 E^{0.12}\right) /\left(0.18+E^{0.12}\right)$, where $E$ is TKE. The coefficient of determination of the fitting is 0.07 .

turbulence (Schmitt 2007; Cava et al. 2012). Thus, the Weibull distribution, that is, the stretchedexponentially truncated power-law distribution, can also be considered as a model of return-time distribution.

(ii) The Weibull distribution (Laherrère and Sornette 1998):

$$
f(\Omega)=k \lambda^{-k} \Omega^{k-1} \exp \left[-(\Omega / \lambda)^{k}\right],
$$

where $\lambda=1 / \Gamma(1+1 / k)$ for $0<k \leq 1$. The tail distribution of the Weibull distribution is

$$
P(\Omega>\omega)=\int_{\omega}^{\infty} f(\Omega) d \Omega=\exp \left[-\left(\frac{\omega}{\lambda}\right)^{k}\right] .
$$

If $k=1$, the Weibull distribution is just the exponential distribution for statistically independent time series.

(iii) The $q$-exponential distribution (Ludescher et al. 2011; Manshour et al. 2016; Carbone et al. 2018):

$$
f(\Omega)=(2-q) \beta[1-(1-q) \beta x]^{1 /(1-q)},
$$

where $\beta=1 /(3-2 q)$ for $1 \leq q<3 / 2$. The tail distribution of the $q$-exponential distribution is

$P(\Omega>\omega)=\int_{\omega}^{\infty} f(\Omega) d \Omega=\left[1-\left(\frac{1}{q^{\prime}}-1\right) \beta \omega\right]^{1 /\left(1-q^{\prime}\right)}$,

where $q^{\prime}=1 /(2-q)$. The $q$-exponential distribution reduces to the exponential distribution as $q \rightarrow 1$.

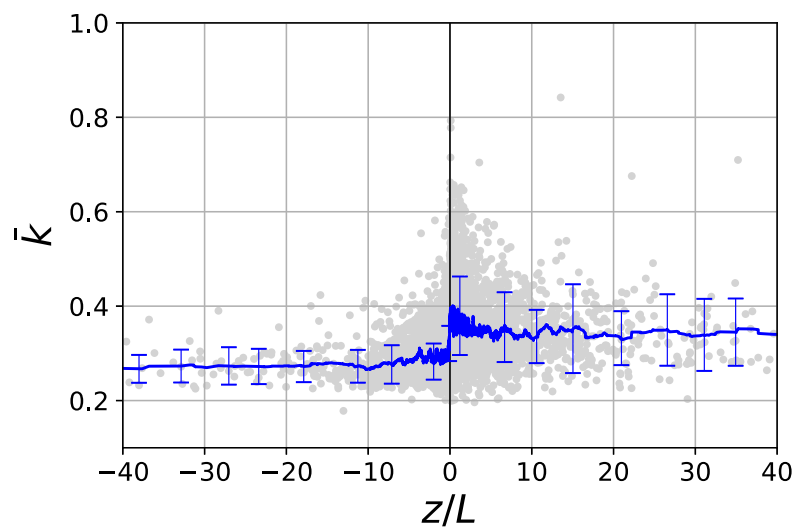

FIG. 12. Dependence of the scale-averaged clusterization intermittency exponent $\bar{k}$ with $z / L$, where $z$ is the observational height and $L$ is the Obukhov length. The exponent for each sample is shown by a gray point, and the moving average along the abscissa with a running window of 50 points is shown by a blue line. The standard deviation of $\bar{k}$ is denoted by an error bar.

Figure 7 shows the tail distributions of return time at scale $\tau=0.05,10$, and $100 \mathrm{~s}$ for case A and case B. The data are fitted to the Weibull distribution, the stretched exponential distribution, and the $q$-exponential distribution, respectively. From this figure, one can see that the $q$-exponential distribution cannot fit the data well at large scales. Both the Weibull distribution and the stretched exponential distribution can fit the data well, but the former is slightly better at large scales. We have analyzed all samples and found that the Weibull distribution is indeed the best one (see Fig. 8).

When $0<k<1$, the Weibull distribution describes the return-time distribution of long-term correlated time series (Blender et al. 2008; Santhanam and Kantz 2008). With decreasing $k$, the Weibull distribution becomes more and more heavy tailed, which indicates that long return time will appear more likely in the telegraphic approximation series, and clusterization intermittency will be more significant (Blender et al. 2015). Therefore, $k$, called the clusterization intermittency exponent, is used to measure clusterization intermittency in the following analysis.

\section{Characteristics of clusterization intermittency}

In this section, we present the characteristics of clusterization intermittency of turbulent velocity fluctuations in the atmospheric boundary layer. As discussed in the above section, the clusterization intermittency is measured by the clusterization intermittency exponent $k$, defined by the shape parameter of the 
(a) 2009-09-11 13:30:00
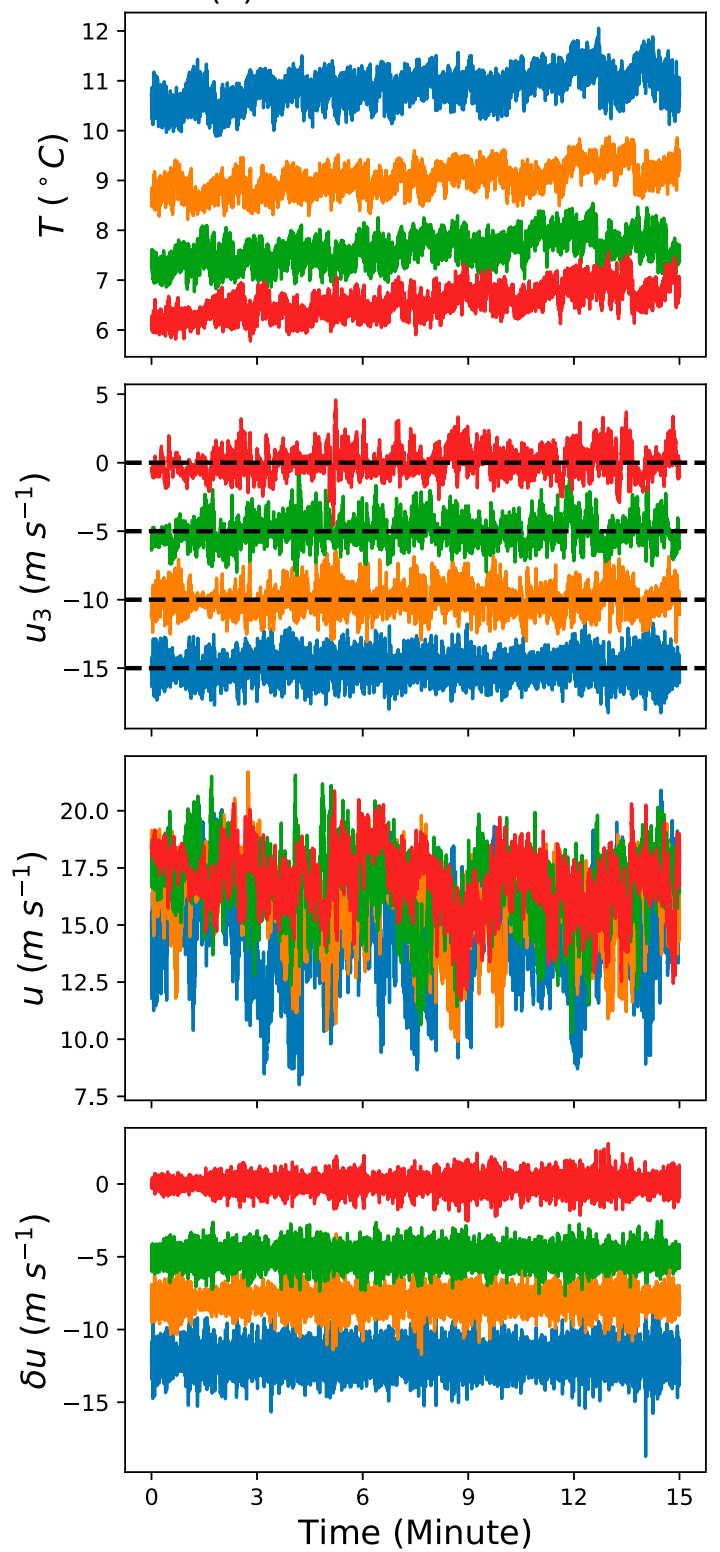

(b) 2009-09-18 12:45:00
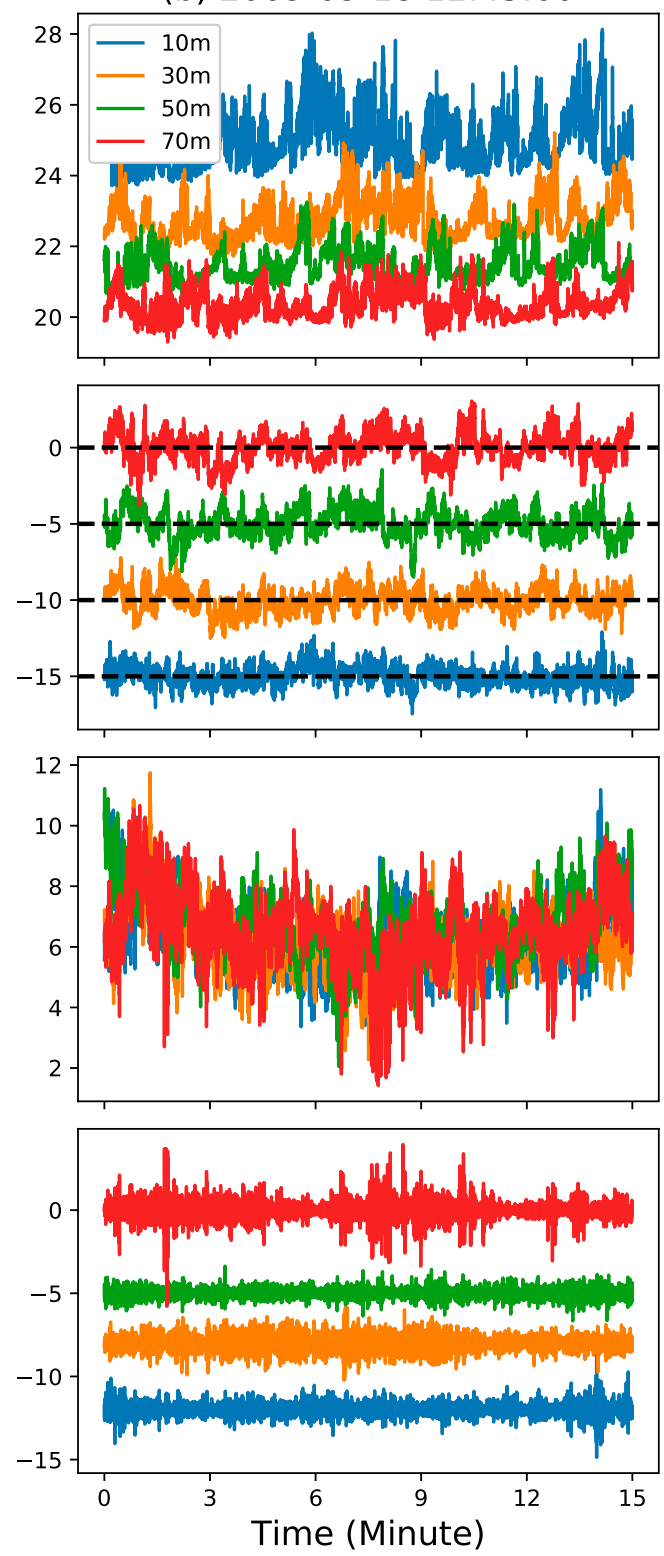

FIG. 13. Time series of (top to bottom) temperature $T$, vertical velocity $u_{3}$, wind speed $u$, and wind speed increment $\delta u$ with a time lag of $0.05 \mathrm{~s}$ in (a) the nearly neutral boundary layer (the Obukhov length $L=-476.98 \mathrm{~m}$ ) and (b) the convective boundary layer $(L=-11.57 \mathrm{~m})$. For clarity, the temperature series at 30,50 , and $70 \mathrm{~m}$ are shifted downward by $1^{\circ}, 2^{\circ}$, and $3^{\circ} \mathrm{C}$, respectively. The vertical velocity series at 10,30 , and $50 \mathrm{~m}$ are shifted downward by 15,10 , and $5 \mathrm{~m} \mathrm{~s}^{-1}$, respectively. The wind speed increment series at 10,30 , and $50 \mathrm{~m}$ are shifted downward by 12,8 , and $5 \mathrm{~m} \mathrm{~s}^{-1}$, respectively. The wind speed series are not shifted. The broken lines in the plots of $u_{3}$ mark the zero vertical velocity before shifting.

Weibull distribution. The smaller $k$ means more significant clusterization intermittency.

\section{a. Diurnal variation}

For case $\mathrm{A}$ and case $\mathrm{B}$, the exponent $k$ is plotted as a function of scale $\tau$ shown in Fig. 9a, where it is found that $k$ decreases with increasing scale, and at small scales, $k$ decreases logarithmically with scale. We have performed the similar analysis on all samples and found that the clusterization intermittency exponent has a decreasing trend with increasing scale (see Fig. 9b). This indicates that the clusterization intermittency of turbulent velocities is normally stronger at larger scales, contrary to the magnitude intermittency that is normally 

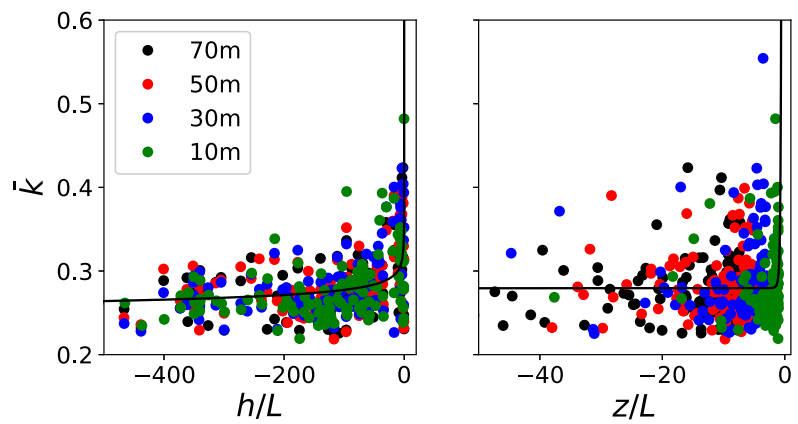

FIG. 14. Dependence of scaled-averaged clusterization intermittency exponent $\bar{k}$ with (left) $h / L$ and (right) $z / L$, where $L$ is the Obukhov length, $h$ is the boundary layer height, and $z$ is the observational height. The curves show the nonlinear least squares fittings with data.

stronger at smaller scales. To estimate the overall clusterization intermittency for a sample, we average the clusterization intermittency exponent $k$ over scales between $\tau_{1}$ and $\tau_{2}$ :

$$
\bar{k}=\frac{1}{\tau_{2}-\tau_{1}} \int_{\tau_{1}}^{\tau_{2}} k(\tau) d \tau,
$$

where $\tau_{1}$ and $\tau_{2}$ are respectively set to the minimum $(0.05 \mathrm{~s})$ and maximum scales $(204.8 \mathrm{~s})$ shown in Fig. 9.

The time variation of $\bar{k}$ at $10,30,50$, and $70 \mathrm{~m}$ is shown in Fig. 10. From this figure, significant diurnal variation of clusterization intermittency is found at different heights. Normally, $\bar{k}$ is smaller in daytime and larger in nighttime, which indicates that clusterization intermittency is more significant in daytime than in nighttime. The buoyancy is usually help for turbulence generation in daytime but usually depress turbulence generation in nighttime. The turbulence kinetic energy (TKE) is typically larger in daytime (Stull 1988). According to the K41 theory, the more energy turbulence extracts from largescale eddies, the more energy finescale eddies gain in the cascade process before energy dissipates (Kolmogorov 1941). The gained energy could entail the presence of finescale coherent structures, such as vortex tubes and strain sheets (Wilczek 2015; Dong et al. 2020). Thus, clusterization intermittency manifesting itself in finescale coherent structures is typically larger in daytime than in nighttime (Fig. 10), and is more likely to be large with larger TKE (Fig. 11). On the other hand, $\bar{k}$ at $10 \mathrm{~m}$ is generally smaller than that at higher height. In the turbulent boundary layer, it is a well-known fact that the characteristic scale and energy of turbulent eddies grow with height (Jiménez 2018). The smaller and weaker turbulent eddies near wall would dissipate much faster than those in higher levels, and lesser energy is passed to finescale eddies in the cascade process to entail the

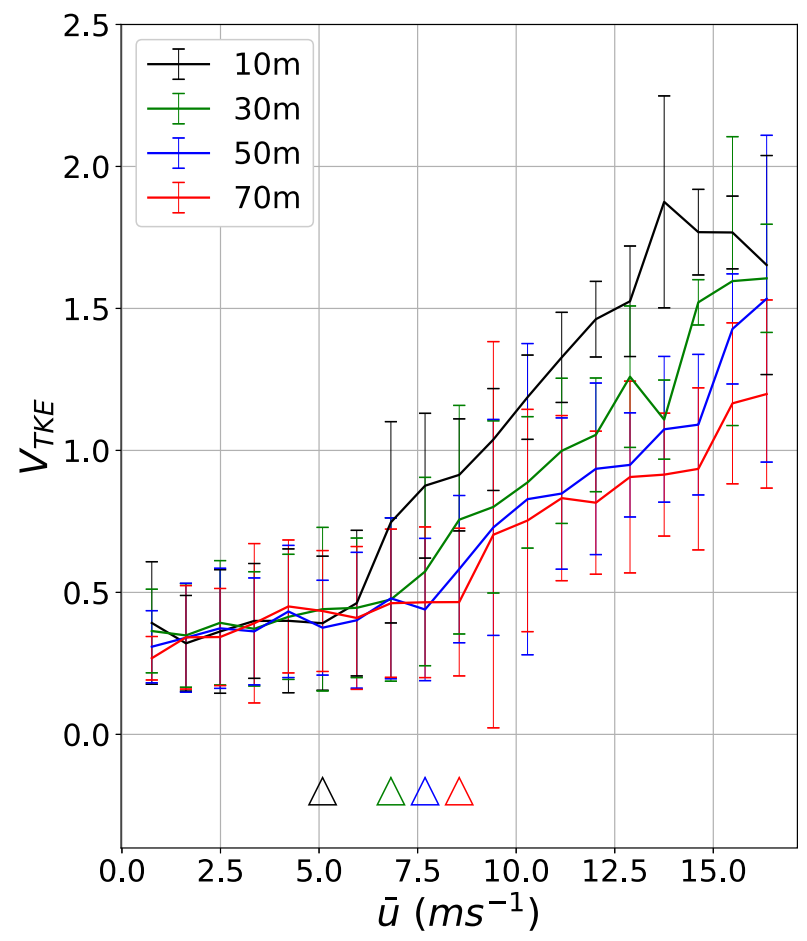

FIG. 15. The relationship between the bin-averaged root square of turbulence kinetic energy $V_{\text {TKE }}$ and the mean wind speed $\bar{u}$ at the four observational heights. The standard deviation of $V_{\mathrm{TKE}}$ within each bin of $\bar{u}$ is denoted by an error bar. The threshold wind speed at each observation height is denoted by a triangle in the same color as the height.

presence of coherent structures. This could lead to weaker clusterization intermittency near surface.

\section{b. Dependence with stability}

The diurnal variation indicates that clusterization intermittency is related to boundary layer stability. Here, we compute the variation of $\bar{k}$ with the stability parameter $z / L$, where $z$ is the observation height and $L$ is the Obukhov length. The results are shown in Fig. 12, where it is found that $\bar{k}$ is normally smaller in the unstable boundary layer than in the stable boundary layer, and the clusterization intermittency is thus more significant under unstable conditions. A similar result is also found for the intermittent heat flux (Katul et al. 1994). In the unstable boundary layer, a dropping trend of $\bar{k}$ is observed from neutral to unstable condition and then $\bar{k}$ is almost constant. In the stable boundary layer, data are very scattered, and we cannot find an evident trend of $\bar{k}$.

The variations of $\bar{k}$ with height are analyzed over each 15 -min epoch. The standard deviation of $\bar{k}$ over the four observational heights is computed by

$$
\mathrm{SD}[\bar{k}]=\sqrt{\frac{1}{4} \sum_{n=1}^{4}\left(\bar{k}_{z_{n}}-\langle\bar{k}\rangle_{z}\right)^{2}}
$$


TABLE 2. Mean wind speed $u$ and turbulence kinetic energy (TKE) for cases in the regime 1 and regime 2, respectively. The threshold wind speed from regime 1 to regime 2 is also listed.

\begin{tabular}{|c|c|c|c|c|c|}
\hline \multirow[b]{2}{*}{ Height (m) } & \multicolumn{2}{|c|}{ Case in regime 1} & \multicolumn{2}{|c|}{ Case in regime 2} & \multirow[b]{2}{*}{ Threshold $u\left(\mathrm{~m} \mathrm{~s}^{-1}\right)$} \\
\hline & $\overline{\bar{u}\left(\mathrm{~m} \mathrm{~s}^{-1}\right)}$ & $\operatorname{TKE}\left(\mathrm{m}^{2} \mathrm{~s}^{-2}\right)$ & $\bar{u}\left(\mathrm{~m} \mathrm{~s}^{-1}\right)$ & $\operatorname{TKE}\left(\mathrm{m}^{2} \mathrm{~s}^{-2}\right)$ & \\
\hline 70 & 2.20 & 0.05 & 9.85 & 3.45 & 8.56 \\
\hline 50 & 1.64 & 0.15 & 9.45 & 3.16 & 7.69 \\
\hline 30 & 0.82 & 0.10 & 8.50 & 2.60 & 6.82 \\
\hline 10 & 1.24 & 0.07 & 7.45 & 2.64 & 5.09 \\
\hline
\end{tabular}

where $\bar{k}_{z_{n}}$ is the scale-averaged clusterization intermittency exponent at height $z_{n}$, and $\langle\cdot\rangle_{z}$ denotes the arithmetic mean over the four observational heights. It is found that $75 \% 15$-min samples at daytime have $\mathrm{SD}[\bar{k}]<0.03$ (Fig. 16). The slight variation of $\bar{k}$ with height could be related to nonlocal turbulence generated by bulk buoyancy-driven instability in the very unstable boundary layer or the bulk wind shear in the nearly neutral boundary layer. Figure 13 shows two cases in the very unstable and the nearly neutral situations, respectively. In the very unstable case, ramp structures in the temperature time series (see $T$ in Fig. 13b) and large updrafts and downdrafts in the vertical velocity time series (see $u_{3}$ in Fig. 13b) are frequently observed, when thermals pass the observational tower (Garratt 1992). In the nearly neutral case, there are no evident ramp structures in the temperature time series (see $T$ in Fig. 13a) and updrafts and downdrafts appear less frequently in the vertical velocity time series (see $u_{3}$ in Fig. 13a). For the two cases, the clusterization intermittency is evident at all heights (see $\delta u_{3}$ in Fig. 13), and $\bar{k}$ is found to vary slightly along height (see the black and green lines in Fig. 18). The values of $\operatorname{SD}[\bar{k}]$ in both cases are smaller than 0.02 .

The fact that $\bar{k}$ varies slightly with height indicates that observation height is not a very relevant parameter in the similarity analysis of clusterization intermittency in cases with nonlocal flow instabilities. In the convective surface layer, the Monin-Obukhov similarity is usually replaced by the mixing-layer similarity, where the observational height $z$ is replaced by the boundary layer height $h$ (Panofsky et al. 1977; Holtslag and Nieuwstadt 1986). We analyze the data with $z / L<-1$ and find that the dependence of clusterization intermittency exponent with $h / L$ is less scattered than that with $z / L$ (Fig. 14). The boundary layer height is estimated by the wavelength of maximum energy in the vertical velocity spectrum (Kaimal et al. 1982; Liu et al. 2011). The data in Fig. 14 are fitted with a power function by using the nonlinear least squares method. The fitting results show that

$$
\bar{k}\left(\frac{h}{L}\right)=0.17\left(\frac{0.17}{-h / L}\right)^{0.10}+0.19
$$

and

$$
\bar{k}\left(\frac{z}{L}\right)=0.54\left(\frac{0.54}{-z / L}\right)^{5.43}+0.28
$$

The coefficients of determination of the fittings with Eqs. (26) and (27) are 0.21 and 0.006, respectively

\section{c. The hockey-stick theory}

The turbulence characteristics in the nocturnal stable boundary layer are usually analyzed by separating between two regimes, typically denoted as weakly stable and very stable (Mahrt 2014). One approach to delineate regimes is based on the hockey-stick theory (Sun et al. 2012). The theory states that the two regimes could be distinguished by the relationship between mean wind speed $\bar{u}$ and square root of turbulence kinetic energy $V_{\mathrm{TKE}}$. The very stable regime where $V_{\mathrm{TKE}}$ is weak and increases slightly with $\bar{u}$ is called regime 1 and the weakly stable regime where $V_{\mathrm{TKE}}$ increases rapidly with $\bar{u}$ after $\bar{u}$ exceeds a threshold value is called regime 2 . The relationship between $V_{\mathrm{TKE}}$ and $\bar{u}$ of data from 1900

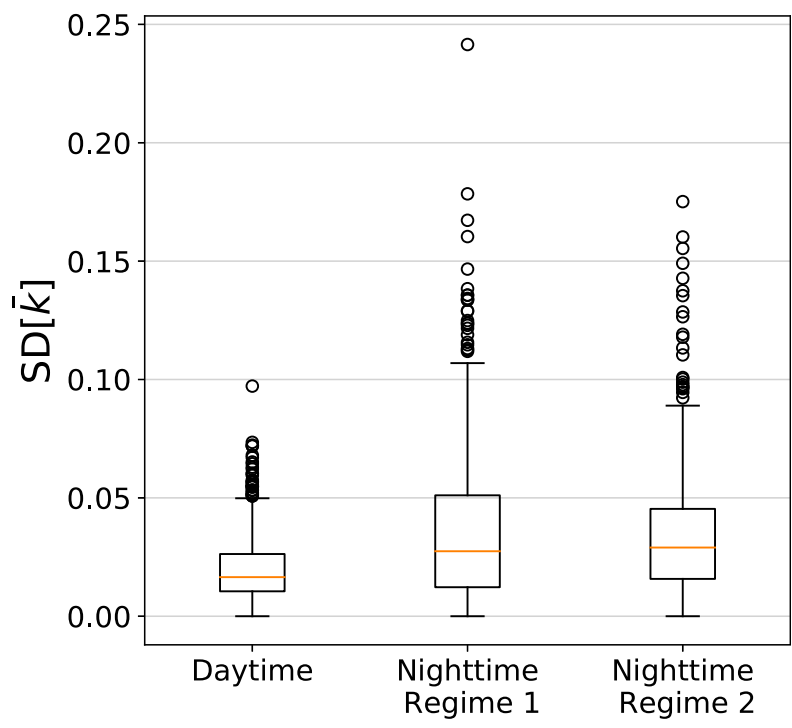

FIG. 16. A boxplot of standard deviation of scale-averaged clusterization intermittency $\bar{k}$ over height in daytime and in regime 1 and regime 2 in nighttime. 
(a) Regime 1: 2009-09-28 00:30:00

(b) Regime 2: 2009-09-01 04:00:00
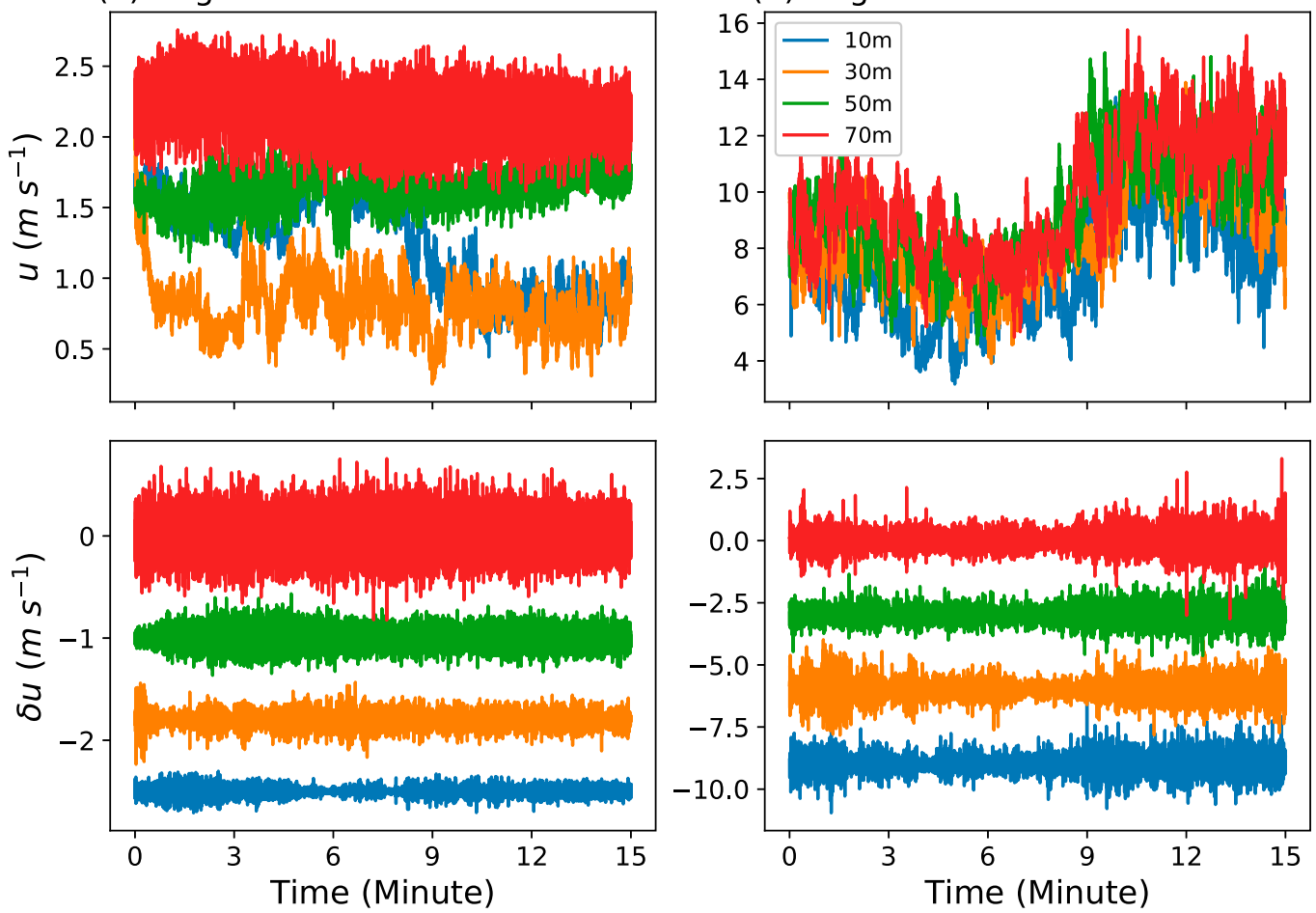

FIG. 17. Time series of (top) wind speed $u$ and (bottom) wind speed increment $\delta u$ with a time lag of $0.05 \mathrm{~s}$ in (a) regime 1 and (b) regime 2 in nighttime. For clarity, the wind speed increment series in regime 1 at 10, 30, and $50 \mathrm{~m}$ are shifted downward by $2.5,1.8$, and $1 \mathrm{~m} \mathrm{~s}^{-1}$, respectively. The wind speed increment series in regime 2 at 10 , 30 , and $50 \mathrm{~m}$ are shifted downward by 9,6 , and $3 \mathrm{~m} \mathrm{~s}^{-1}$, respectively. The wind speed series are not shifted.

to 0600 LST is shown in Fig. 15, and the threshold velocity is listed in Table 2 . The two regimes can be clearly observed at all heights, and the threshold wind speed increases with height, as found in Sun et al. (2012).

The variation of $\bar{k}$ with height is analyzed in the two regimes. It is found that $\bar{k}$ varies more slightly in regime 2 than in regime 1 , where $75 \% \mathrm{SD}[\bar{k}]$ in regime 1 are larger than that in regime 2 , and very large $\mathrm{SD}[\bar{k}]$ are more likely to appear in regime 1 (Fig. 16). The results coincide with the hockey-stick theory. According to this theory, turbulence in regime 1 is mainly generated by local wind shear and occurs frequently at some specific heights, while turbulence in regime 2 is mainly generated by bulk wind shear and occurs at most of observational heights (Sun et al. 2012).

Two cases in regime 1 and regime 2 are shown in Figs. $17 \mathrm{a}$ and $17 \mathrm{~b}$, respectively. In the case of regime 2, one can see that clusterization intermittency is evident at the four heights (see $\delta u$ in Fig. 17b), and $\bar{k}$ varies slightly with heights (see the blue line in Fig. 18). In the case of regime 1 , clusterization intermittency is evident at the height of 10 and $30 \mathrm{~m}$ (see $\delta u$ in Fig. 17a). This could be related to the occurrence of jet-like wind shear below $30 \mathrm{~m}$ (see $u$ in Fig. 17), as found in (Cava et al. 2019).
At higher levels, the time series look more like Gaussian white noises. In this case, $\bar{k}$ is smaller at 10 and $30 \mathrm{~m}$ and becomes larger at higher heights (see the red line in Fig. 18). This example shows the possibility of the link between the jet-like event and clusterization intermittency. A better understanding of this link and the height dependence of clusterization intermittency would be obtained in the future research.

\section{Conclusions}

In this paper, we analyzed the multiscale clusterization intermittency of atmospheric turbulence by using a $20 \mathrm{~Hz}$ ultrasonic dataset lasting for one month. The turbulent velocity fluctuations at different scales, defined by the absolute velocity increments at different time lags, is found to be long-term correlated in statistics. By the fluctuation analysis, the clusterization intermittency, which refers to the fact that large velocity increments tend to appear in clusters, is found to be related to the long-term correlation. This finding reveals that clusterization intermittency is different to magnitude intermittency: the latter is related to non-Gaussianity, 


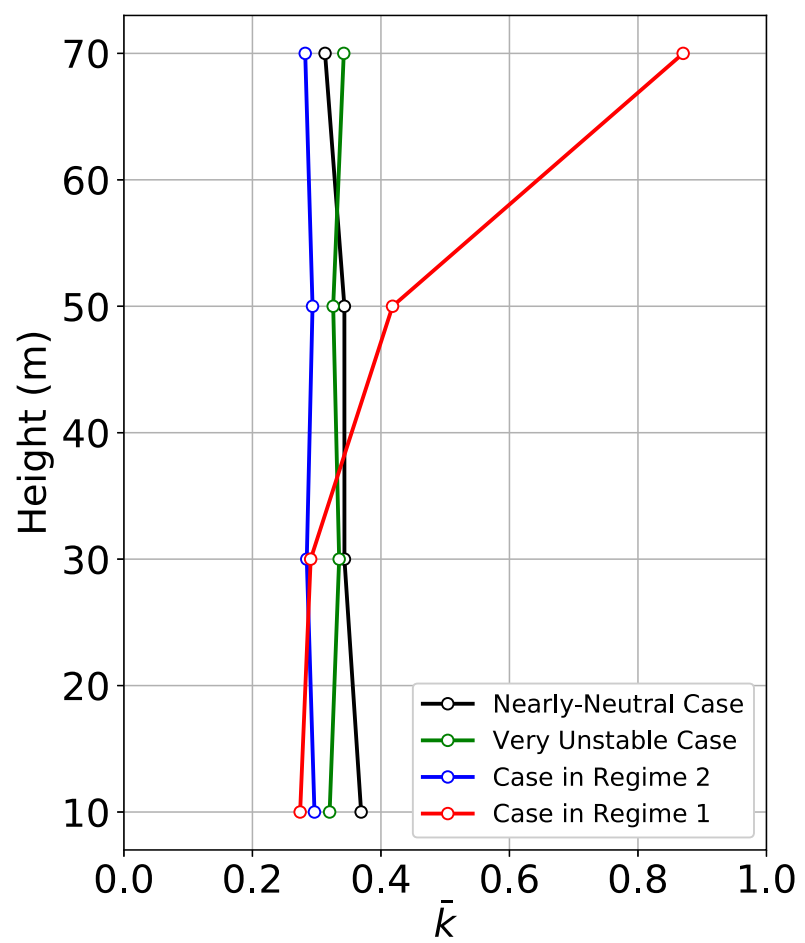

FIG. 18. Variations of $\bar{k}$ with height for cases shown in Fig. 13a (black line), Fig. 13b (green line), Fig. 17a (red line), and Fig. 17b (blue line).

while the former is not related to non-Gaussianity. Even the non-Gaussian random process, for example, the fractional Brownian motion (Mandelbrot and Ness 1968; Ding and Yang 1995), may have clusterization intermittency. Thus, the magnitude intermittency exponent cannot be used to measure clusterization intermittency.

Basing on the return-time statistics, we propose a new exponent to measure clusterization intermittency. The return-time statistics include return-time distributions of the telegraphic approximation of absolute velocity increments and the corresponding randomly shuffled increments respectively at different scales. Comparing the return-time distribution of shuffled increments with the exponential distribution, that is strictly proved to be true for any statistically independent time series, one can set an optimal threshold in the telegraphic approximation. For the return-time distribution of raw increments, we found that it can be well fitted by the Weibull distribution. The shape parameter of the Weibull distribution describes the occurrence probability of long return times, and thus can be used to measure clusterization intermittency. The observations of this parameter for all samples indicate the following: 1) Contrary to magnitude intermittency, clusterization intermittency weakens as scale decreases; 2) diurnal variation of clusterization intermittency is evident at different heights. Normally, the intermittency strengthens at daytime and weakens at nighttime; 3) clusterization intermittency is normally stronger in the unstable boundary layer than in the stable boundary layer. In the very unstable boundary layer, clusterization intermittency scales better by the mixedlayer similarity, where the boundary layer height replaces the observational height in the Monin-Obukhov similarity; 4) according to the hockey-stick theory, data in the nocturnal stable boundary layer are divided into two regimes. Clusterization intermittency varies more slightly with height in the regime with large mean wind speed than in the regime with small mean wind speed. The results coincide with the hockey-stick theory, where turbulence in the regime with large mean wind speed is mainly generated by bulk wind shear, and turbulence in the regime with small mean wind speed is mainly generated by local wind shear.

Our work indicates that finescale clusterization intermittency is intimately related to the boundary layer instability, which transfers energy from large-scale motions to finescale motions in the cascade process. In the future, we will combine data analysis and numerical model to build up some possible connections between large-scale motions and clusterization intermittency. This will be helpful to understand the nature of clusterization intermittency and design new parameterizations of intermittent turbulence to improve numerical model (i.e., large-eddy simulation) of boundary layer motions.

Acknowledgments. This work is supported by the National Nature Science Foundation of China (41675012) and the National Key Research and Development Program of China (2016YFC0208802 and 2017YFC0209605).

\section{REFERENCES}

Altmann, E. G., and H. Kantz, 2005: Recurrence time analysis, long-term correlations, and extreme events. Phys. Rev. E, 71, 056106, https://doi.org/10.1103/PhysRevE.71.056106.

Bacry, E., and J. F. Muzy, 2003: Log-infinitely divisible multifractal processes. Commun. Math. Phys., 236, 449-475, https://doi.org/ 10.1007/s00220-003-0827-3.

_ J. Delour, and J. F. Muzy, 2001: Multifractal random walk. Phys. Rev. E, 64, 026103, https://doi.org/10.1103/PhysRevE.64.026103.

Baile, R., J. F. Muzy, and P. Poggi, 2011: Short-term forecasting of surface layer wind speed using a continuous random cascade model. Wind Energy, 14, 719-734, https://doi.org/10.1002/we.452.

Beran, J., 1994: Statistics for Long-Memory Processes. Chapman and Hall, 315 pp.

Bershadskii, A., J. J. Niemela, A. Praskovsky, and K. R. Sreenivasan, 2004: "Clusterization" and intermittency of temperature fluctuations in turbulent convection. Phys. Rev. E, 69, 056314, https://doi.org/10.1103/PhysRevE.69.056314.

Blender, R., K. Fraedrich, and F. Sienz, 2008: Extreme event return times in long-term memory processes near $1 / \mathrm{f}$. 
Nonlinear Processes Geophys., 15, 557-565, https://doi.org/ 10.5194/npg-15-557-2008.

_ C. C. Raible, and F. Lunkeit, 2015: Non-exponential return time distributions for vorticity extremes explained by fractional Poisson processes. Quart. J. Roy. Meteor. Soc., 141, 249-257, https://doi.org/10.1002/qj.2354.

Boettcher, F., C. Renner, H.-P. Waldl, and J. Peinke, 2003: On the statistics of wind gusts. Bound.-Layer Meteor., 108, 163-173, https://doi.org/10.1023/A:1023009722736.

Bos, W. J. T., L. Liechtenstein, and K. Schneider, 2007: Small-scale intermittency in anisotropic turbulence. Phys. Rev. E, 76, 046310, https://doi.org/10.1103/PhysRevE.76.046310.

Bou-Zeid, E. R., C. Higgins, H. Huwald, C. Meneveau, and M. B. Parlange, 2010: Field study of the dynamics and modelling of subgrid-scale turbulence in a stable atmospheric surface layer over a glacier. J. Fluid Mech., 665, 480-515, https://doi.org/ 10.1017/S0022112010004015.

Bunde, A., J. F. Eichner, J. W. Kantelhardt, and S. Havlin, 2005: Long-term memory: A natural mechanism for the clustering of extreme events and anomalous residual times in climate records. Phys. Rev. Lett., 94, 048701, https://doi.org/10.1103/ PhysRevLett.94.048701.

Calif, R., and F. G. Schmitt, 2012: Modeling of atmospheric wind speed sequence using a lognormal continuous stochastic equation. J. Wind Eng. Ind. Aerodyn., 109, 1-8, https://doi.org/ 10.1016/j.jweia.2012.06.002.

Carbone, F., and Coauthors, 2018: The superstatistical nature and interoccurrence time of atmospheric mercury concentration fluctuations. J. Geophys. Res. Atmos., 123, 764-774, https:// doi.org/10.1002/2017JD027384.

Castaing, B., Y. Gagne, and E. J. Hopfinger, 1990: Velocity probability density functions of high Reynolds number turbulence. Physica D, 46, 177-200, https://doi.org/10.1016/0167-2789(90) 90035-N.

Cava, D., and G. G. Katul, 2009: The effects of thermal stratification on clustering properties of canopy turbulence. Bound-Layer Meteor., 130, 307-325, https://doi.org/10.1007/s10546-008-9342-6.

, - - A. Molini, and C. Elefante, 2012: The role of surface characteristics on intermittency and zero-crossing properties of atmospheric turbulence. J. Geophys. Res., 117, D01104, https://doi.org/10.1029/2011JD016167.

_- L. Mortarini, U. Giostra, O. Acevedo, and G. Katul, 2019: Submeso motions and intermittent turbulence across a nocturnal low-level jet: A self-organized criticality analogy. Bound.-Layer Meteor., 172, 17-43, https://doi.org/10.1007/ s10546-019-00441-8.

Ding, M., and W. Yang, 1995: Distribution of the first return time in fractional Brownian motion and its application to the study of on-off intermittency. Phys. Rev. E, 52, 207-213, https://doi.org/ 10.1103/physreve.52.207.

Dong, S., Y. Huang, X. Yuan, and A. Lozano-Durán, 2020: The coherent structure of the kinetic energy transfer in shear turbulence. J. Fluid Mech., 892, A22, https://doi.org/10.1017/ jfm.2020.195.

Duman, T., A. Trakhtenbrot, D. Poggi, M. Cassiani, and G. G. Katul, 2016: Dissipation intermittency increases long-distance dispersal of heavy particles in the canopy sublayer. Bound.-Layer Meteor., 159, 41-68, https://doi.org/10.1007/s10546-015-0112-y.

Eichner, J. F., J. W. Kantelhardt, A. Bunde, and S. Havlin, 2007: Statistics of return intervals in long-term correlated records. Phys. Rev. E, 75, 011128, https://doi.org/10.1103/PhysRevE.75.011128.

Franzke, C. L. E., T. Graves, N. W. Watkins, R. B. Gramacy, and C. Hughes, 2012: Robustness of estimators of long-range dependence and self-similarity under non-Gaussianity. Philos. Trans. Roy. Soc., 370A, 1250-1267, https://doi.org/10.1098/ rsta.2011.0349.

Friedrich, R., and J. Peinke, 1997: Description of a turbulent cascade by a Fokker-Planck equation. Phys. Rev. Lett., 78, 863-866, https://doi.org/10.1103/PhysRevLett.78.863.

Frisch, U., 1995: Turbulence: The Legacy of A. N. Kolmogorov. Cambridge University Press, 296 pp.

Garratt, J. R., 1992: The Atmospheric Boundary Layer. Cambridge University Press, $316 \mathrm{pp}$.

Granero-Belinchón, C., S. G. Roux, and N. B. Garnier, 2018: Kullback-Leibler divergence measure of intermittency: Application to turbulence. Phys. Rev. E, 97, 013107, https:// doi.org/10.1103/PhysRevE.97.013107.

Holtslag, A. A. M., and F. T. M. Nieuwstadt, 1986: Scaling the atmospheric boundary layer. Bound.-Layer Meteor., 36, 201-209, https://doi.org/10.1007/BF00117468.

Jiménez, J., 2018: Coherent structures in wall-bounded turbulence. J. Fluid Mech., 842, 1, https://doi.org/10.1017/jfm.2018.144.

Kaimal, J. C., and J. J. Finnigan, 1994: Atmospheric Boundary Layer Flows: Their Structure and Measurement. Oxford University Press, 289 pp.

, and Coauthors, 1982: Estimating the depth of the daytime convective boundary layer. J. Appl. Meteor., 21, 1123-1129, https:// doi.org/10.1175/1520-0450(1982)021<1123:ETDOTD>2.0.CO;2.

Kantelhardt, J. W., E. Koscielny-Bunde, H. H. A. Rego, S. Havlin, and A. Bunde, 2001: Detecting long-range correlations with detrended fluctuation analysis. Physica A, 295, 441-454, https://doi.org/10.1016/S0378-4371(01)00144-3.

Karlin, S., and H. M. Taylor, 1975: A First Course in Stochastic Processes. 2nd ed. Academic Press, 557 pp.

Katul, G. G., J. Albertson, M. Parlange, C.-R. Chu, and H. Stricker, 1994: Conditional sampling, bursting, and the intermittent structure of sensible heat flux. J. Geophys. Res., 99, 22 869-22 876, https://doi.org/10.1029/94JD01679.

Kit, E., C. M. Hocut, D. Liberzon, and H. J. S. Fernando, 2017: Fine-scale turbulent bursts in stable atmospheric boundary layer in complex terrain. J. Fluid Mech., 833, 745-772, https:// doi.org/10.1017/jfm.2017.717.

Kolmogorov, A. N., 1941: The local structure of turbulence in incompressible viscous fluid for very large Reynolds numbers. Dokl. Akad. Nauk SSSR, 30, 299-303.

Kratz, M. F., 2006: Level crossings and other level functionals of stationary Gaussian processes. Probability Surveys, 3, 230-288, https://doi.org/10.1214/154957806000000087.

Laherrère, J., and D. Sornette, 1998: Stretched exponential distributions in nature and economy: "fat tails" with characteristic scales. Eur. Phys. J., 2B, 525-539, https://doi.org/10.1007/ s100510050276.

Lauren, M. K., M. Menabde, A. W. Seed, and G. L. Austin, 1999: Characterisation and simulation of the multiscaling properties of the energy-containing scales of horizontal surface-layer winds. Bound.-Layer Meteor., 90, 21-46, https://doi.org/10.1023/A: 1001749126625.

Li, Q., and Z. Fu, 2013: The effects of non-stationarity on the clustering properties of the boundary-layer vertical wind velocity. Bound.-Layer Meteor., 149, 219-230, https://doi.org/ 10.1007/s10546-013-9840-z.

Liu, L., and F. Hu, 2013: Cascade-like and scaling behavior of wind velocity increments in the atmospheric surface layer. Physica A, 392, 5808-5816, https://doi.org/10.1016/j.physa.2013.07.054. , — - X.-L. Cheng, and L.-L. Song, 2010: Probability density functions of velocity increments in the atmospheric boundary 
layer. Bound.-Layer Meteor., 134, 243-255, https://doi.org/ 10.1007/s10546-009-9441-z.

,-- , and,- 2011 : Probability density functions of turbulent velocity and temperature fluctuations in the unstable atmospheric surface layer. J. Geophys. Res., 116, D12117, https://doi.org/10.1029/2010JD015503.

,-- , and,- 2014 : Extreme fluctuations of vertical velocity in the unstable atmospheric surface layer. Nonlinear Processes Geophys., 21, 463-475, https://doi.org/10.5194/npg-21-463-2014. ,-- , and S. Huang, 2019: A multifractal random-walk description of atmospheric turbulence: Small-scale multiscaling, long-tail distribution, and intermittency. Bound.-Layer Meteor., 172, 351-370, https://doi.org/10.1007/s10546-019-00451-6.

Ludescher, J., C. Tsallis, and A. Bunde, 2011: Universal behaviour of interoccurrence times between losses in financial markets: An analytical description. Europhys. Lett., 95, 68002, https:// doi.org/10.1209/0295-5075/95/68002.

Lyu, R., F. Hu, L. Liu, J. Xu, and X. Cheng, 2018: High-order statistics of temperature fluctuations in an unstable atmospheric surface layer over grassland. Adv. Atmos. Sci., 35, 1265-1276, https://doi.org/10.1007/s00376-018-7248-x.

Mahrt, L., 1999: Stratified atmospheric boundary layers. Bound.-Layer Meteor., 90, 375-396, https://doi.org/10.1023/A:1001765727956.

_ 2014: Stability stratified atmospheric boundary layers. Annu. Rev. Fluid Mech., 46, 23-45, https://doi.org/10.1146/annurevfluid-010313-141354.

Malécot, Y., C. Auriault, H. Kahalerras, Y. Gagne, O. Chanal, B. Chabaud, and B. Castaing, 2000: A statistical estimator of turbulence intermittency in physical and numerical experiments. Eur. Phys. J., 16B, 549-561, https://doi.org/10.1007/ s100510070216.

Mandelbrot, B. B., and J. W. V. Ness, 1968: Fractional Brownian motions, fractional noises and applications. SIAM Rev., 10, 422-437, https://doi.org/10.1137/1010093.

Manshour, P., M. Anvari, N. Reinke, M. Sahimi, and M. R. R. Tabar, 2016: Interoccurrence time statistics in fully-developed turbulence. Sci. Rep., 6, 27452, https://doi.org/10.1038/srep27452.

Mantegna, R. N., and H. E. Stanley, 1999: Introduction to Econophysics: Correlations and Complexity in Finance. Cambridge University Press, 148 pp.

Mortarini, L., D. Cava, U. Giostra, O. Acevedo, L. G. Nogueira Martins, P. E. Soares de Oliveira, and D. Anfossi, 2018: Observations of submeso motions and intermittent turbulent mixing across a low level jet with a 132-m tower. Quart. J. Roy. Meteor. Soc., 144, 172-183, https://doi.org/10.1002/qj.3192.

Muzy, J.-F., and E. Bacry, 2002: Multifractal stationary random measures and multifractal random walks with log infinitely divisible scaling laws. Phys. Rev. E, 66, 056121, https://doi.org/ 10.1103/PhysRevE.66.056121.

Noullez, A., G. Wallace, W. Lempert, R. B. Miles, and U. Frisch, 2000: Transverse velocity increments in turbulent flow using the relief technique. J. Fluid Mech., 339, 287-307, https:// doi.org/10.1017/S0022112097005338.

Panofsky, H. A., H. Tennekes, D. H. Lenschow, and J. C. Wyngaard, 1977: The characteristics of turbulent velocity components in the surface layer under convective conditions. Bound.-Layer Meteor., 11, 355-361, https://doi.org/10.1007/ BF02186086.

Peng, C. K., S. V. Buldyrev, A. L. Goldberger, S. Havlin, F. Sciortino, M. Simons, and H. E. Stanley, 1992: Long-range correlations in nucleotide sequences. Nature, 356, 168-170, https://doi.org/10.1038/356168a0.
Santhanam, M. S., and H. Kantz, 2008: Return interval distribution of extreme events and long-term memory. Phys. Rev. E, 78, 051113, https://doi.org/10.1103/PhysRevE.78.051113.

Schertzer, D., S. Lovejoy, F. Schmitt, Y. Chigirinskaya, and D. Marsan, 1997: Multifractal cascade dynamics and turbulent intermittency. Fractals, 5, 427-471, https://doi.org/10.1142/ S0218348X97000371.

Schmitt, F. G., 2007: Gusts in intermittent wind turbulence and the dynamics of their recurrent times. Wind Energy: Proceedings of the Euromech Colloquium, J. Peinke et al., Eds., Springer, 73-79.

_ D. Schertzer, S. Lovejoy, and Y. Brunet, 1994: Empirical study of multifractal phase transitions in atmospheric turbulence. Nonlinear Processes Geophys., 1, 95-104, https://doi.org/10.5194/ npg-1-95-1994.

Slud, E., 1994: MWI expansions for functionals related to levelcrossing counts. Chaos Expansions, Multiple Wiener-Ito Integrals, and Their Applications. CRC Press, 125-143.

Sreenivasan, K. R., and R. A. Antonia, 1997: The phenomenology of small-scale turbulence. Annu. Rev. Fluid Mech., 29, 435-472, https://doi.org/10.1146/annurev.fluid.29.1.435.

_ , and A. Bershadskii, 2006: Clustering properties in turbulent signals. J. Stat. Phys., 125, 1141-1153, https://doi.org/10.1007/ s10955-006-9112-0.

Stull, R. B., 1988: An Introduction to Boundary Layer Meteorology. Kluwer Academic, 666 pp.

Sun, J., L. Mahrt, R. M. Banta, and Y. L. Pichugina, 2012: Turbulence regimes and turbulence intermittency in the stable boundary layer during CASES-99. J. Atmos. Sci., 69, 338-351, https://doi.org/10.1175/JAS-D-11-082.1.

Tabeling, P., G. Zocchi, F. Belin, J. Maurer, and H. Willaime, 1996: Probability density functions, skewness, and flatness in large Reynolds number turbulence. Phys. Rev. E, 53, 1613, https:// doi.org/10.1103/physreve.53.1613.

Taqqu, M. S., V. Teverovsky, and W. Willinger, 1995: Estimators for long-range dependence: An empirical study. Fractals, 3 , 785-798, https://doi.org/10.1142/S0218348X95000692.

Vercauteren, N., and R. Klein, 2015: A clustering method to characterize intermittent bursts of turbulence and interaction with submesomotions in the stable boundary layer. J. Fluid Mech. 72, 1504-1517, https://doi.org/10.1175/JAS-D-14-0115.1.

Vickers, D., and L. Mahrt, 1997: Quality control and flux sampling problems for tower and aircraft data. J. Atmos. Oceanic Technol., 14, 512-526, https://doi.org/10.1175/1520-0426(1997) $014<0512$ :QCAFSP $>2.0$. CO;2.

Vindel, J. M., and C. Yagüe, 2011: Intermittency of turbulence in the atmospheric boundary layer: Scaling exponents and stratification influence. Bound.-Layer Meteor., 140, 73-85, https://doi.org/ 10.1007/s10546-011-9597-1.

Wächter, M., and Coauthors, 2012: The turbulent nature of the atmospheric boundary layer and its impact on the wind energy conversion process. J. Turbul., 13, N26, https://doi.org/10.1080/ 14685248.2012.696118.

Wei, W., M. Wang, H. Zhang, Q. He, M. Ali, and Y. Wang, 2017: Diurnal characteristics of turbulent intermittency in the Taklimakan Desert. Meteor. Atmos. Phys., 131, 287-297, https://doi.org/10.1007/s00703-017-0572-3.

_, H. Zhang, B. Wu, Y. Huang, X. Cai, Y. Song, and J. Li, 2018: Intermittent turbulence contributes to vertical dispersion of $\mathrm{PM}_{2.5}$ in the North China Plain: Cases from Tianjin. Atmos. Chem. Phys., 18, 12 953-12 967, https://doi.org/10.5194/acp-18-12953-2018.

Wilczek, M., 2015: New insights into the fine-scale structure of turbulence. J. Fluid Mech., 784, 1-4, https://doi.org/10.1017/ jfm.2015.536. 